\title{
A VIEW OF THE NARROW-LINE REGION IN THE INFRARED: ACTIVE GALACTIC NUCLEI WITH RESOLVED FINE-STRUCTURE LINES IN THE SPITZER ARCHIVE
}

\author{
K. M. Dasyra ${ }^{1,2}$, L. C. Ho ${ }^{3}$, H. Netzer ${ }^{4}$, F. Combes $^{2}$, B. Trakhtenbrot ${ }^{4}$, E. Sturm ${ }^{5}$, L. Armus ${ }^{6}$, And D. Elbaz ${ }^{1}$ \\ ${ }^{1}$ Laboratoire AIM, CEA/DSM-CNRS-Université Paris Diderot, Irfu/Service dAstrophysique, CEA Saclay, F-91191 Gif-sur-Yvette, France \\ 2 Observatoire de Paris, LERMA (CNRS:UMR8112), F-75014 Paris, France \\ 3 The Observatories of the Carnegie Institution for Science, Pasadena, CA 91101, USA \\ ${ }^{4}$ School of Physics and Astronomy and the Wise Observatory, Raymond and Beverly Sackler Faculty of Exact Sciences, \\ Tel-Aviv University, Tel-Aviv 69978, Israel \\ ${ }^{5}$ Max-Planck-Institut für Extraterrestrische Physik, 85741 Garching, Germany \\ ${ }^{6}$ Spitzer Science Center, California Institute of Technology, Pasadena, CA 91125, USA \\ Received 2011 March 25; accepted 2011 July 18; published 2011 October 4
}

\begin{abstract}
We queried the Spitzer archive for high-resolution observations with the Infrared Spectrograph of optically selected active galactic nuclei (AGNs) for the purpose of identifying sources with resolved fine-structure lines that would enable studies of the narrow-line region (NLR) at mid-infrared wavelengths. By combining 298 Spitzer spectra with 6 Infrared Space Observatory spectra, we present kinematic information of the NLR for 81, $z \lesssim 0.3$ AGNs. We used the [Ne v], [O IV], [Ne III], and [S IV] lines, whose fluxes correlate well with each other, to probe gas photoionized by the AGN. We found that the widths of the lines are, on average, increasing with the ionization potential of the species that emit them. No correlation of the line width with the critical density of the corresponding transition was found. The velocity dispersion of the gas, $\sigma$, is systematically higher than that of the stars, $\sigma_{*}$, in the AGN host galaxy, and it scales with the mass of the central black hole, $M_{\mathrm{BH}}$. Further correlations between the line widths and luminosities $L$, and between $L$ and $M_{\mathrm{BH}}$, are suggestive of a three-dimensional plane connecting $\log \left(M_{\mathrm{BH}}\right)$ to a linear combination of $\log (\sigma)$ and $\log (L)$. Such a plane can be understood within the context of gas motions that are driven by AGN feedback mechanisms, or virialized gas motions with a power-law dependence of the NLR radius on the AGN luminosity. The $M_{\mathrm{BH}}$ estimates obtained for 35 type 2 AGNs from this plane are consistent with those obtained from the $M_{\mathrm{BH}}-\sigma_{*}$ relation.
\end{abstract}

Key words: galaxies: active - galaxies: kinematics and dynamics - galaxies: nuclei - galaxies: Seyfert - infrared: galaxies - quasars: emission lines

\section{INTRODUCTION}

Meaningful statistical tests of whether the bulk of black hole $(\mathrm{BH})$ growth precedes, is parallel, or follows the peak of star formation activity need to rely on the comparison of several observable parameters as a function of look-back time. In addition to the comparison of the star formation rate (SFR) with the $\mathrm{BH}$ accretion rate (e.g., Marconi et al. 2004; Merloni et al. 2004), the comparison of the stellar mass with the $\mathrm{BH}$ mass, $M_{\mathrm{BH}}$, that is already accumulated at any given redshift $z$ is also desirable. A significant impediment in performing the latter comparison comes from the fact that rapid $\mathrm{BH}$ growth often occurs in highly obscured environments, such as type 2 active galactic nuclei (AGNs) and infrared (IR) bright galaxies. At low $z$ and at low (Seyfert-like) luminosities, $L, 50 \%-70 \%$ of the AGNs are of type 2 (Ho et al. 1997b; Schmitt et al. 2001; Hao et al. 2005). At $z \sim 1$ and at bolometric luminosities that exceed $10^{12} L_{\text {sun }}$, at least 1 out of every 3 AGNs is thought to be of type 2 (Lacy et al. 2007; Gilli et al. 2007).

In type 2 systems, the determination of $M_{\mathrm{BH}}$ is very challenging. Direct methods of measuring $M_{\mathrm{BH}}$, e.g., by spatially resolved sub-parsec or parsec scale kinematics of stars (Genzel et al. 1997), ionized gas (Harms et al. 1994; Macchetto et al. 1997), or water masers (Miyoshi et al. 1995), can only be applicable to very local objects. An alternative method that is successfully applied to type 1 AGNs even at high $z$ (Kaspi et al. 2007) uses the kinematics and the radial extent of gas clouds that are close enough to the $\mathrm{BH}$ to be gravitationally influenced by it. These clouds constitute the AGN broad-line region (BLR). Emission lines tracing the BLR, such as $\mathrm{H} \beta$, $\mathrm{Mg}$ II, and C IV, have typical full width at half maxima (FWHM) exceeding $2000 \mathrm{~km} \mathrm{~s}^{-1}$. The BLR radius is determined by reverberation mapping experiments, which use the natural variability of the AGN continuum and the time-delayed response of the gas (de)excitation to compute the light-travel time across the BLR (Blandford \& McKee 1982; Peterson 1993; Kaspi et al. 2000). Assuming that the clouds are virialized, their distance from the $\mathrm{BH}$ and their velocity dispersion can be used to determine an enclosed dynamical mass and, thus, $M_{\mathrm{BH}}$. However, the method is not applicable to type 2 AGNs where the BLR is obscured.

The lines that are seen in both type 1 and type 2 AGNs are those that typically originate from gas clouds at a few parsecs to a few hundreds of parsecs away from the BH (Netzer 2004; Laor 2007, and references therein). These narrow-line-region (NLR) clouds have FWHM that are typically $\lesssim 1000 \mathrm{~km} \mathrm{~s}^{-1}$. Using the [O III] $5007 \AA$ line, Nelson \& Whittle (1996) demonstrated that the bulk of the gas traced by [O III] has a velocity dispersion that is comparable to that of the stars in the host-galaxy bulge. Subsequently, $M_{\mathrm{BH}}$ was found to scale with the width of the [O III] emission (Nelson 2000; Shields et al. 2003; Greene \& Ho 2005). A similar result was found for the [N II] line at $6583 \AA$ by Ho (2009).

Optical NLR lines can suffer severe obscuration (Kauffmann et al. 2003). This makes IR wavelengths more reliable and often unique for investigating relations between NLR kinematics and $M_{\mathrm{BH}}$. To optimize the use of narrow lines as gravitational potential tracers for obscured AGNs, we performed a similar analysis in mid-infrared (MIR) wavelengths using Spitzer and 
Infrared Space Observatory (ISO) high-resolution spectra. We demonstrated that the widths of the NLR lines [Nev] at $14.32 \mu \mathrm{m}$ and [O IV] at $25.89 \mu \mathrm{m}$ also scale with $M_{\mathrm{BH}}$ (Dasyra et al. 2008). This result could provide a means of probing NLR kinematics and weighing $\mathrm{BH}$ masses in obscured galaxies at high $z$ with the next generation of IR telescopes.

The goal of this paper is to perform an extended study of the NLR kinematics of optically selected type 1 and type 2 AGNs in the MIR using all spectra that are available in the Spitzer archive. We aim to investigate for differences in the gas kinematics as traced by various fine-structure lines to test how the gas velocities compare with $M_{\mathrm{BH}}$, and to further estimate the masses of local obscured AGNs. For all computations, we use a $\Lambda$ CDM cosmology with $H_{0}=70 \mathrm{~km} \mathrm{~s}^{-1} \mathrm{Mpc}^{-1}, \Omega_{m}=0.3$, and $\Omega_{\Lambda}=0.7$.

\section{THE SAMPLE}

To study the NLR gas kinematics as probed by MIR emission lines, we queried the entire Spitzer archive for high-resolution observations of AGNs obtained with the Infrared Spectrograph (IRS; Houck et al. 2004). These spectra have a resolving power of $\sim 500 \mathrm{~km} \mathrm{~s}^{-1}$, sufficient for resolving features in the NLR of several local AGNs (Dasyra et al. 2008). We downloaded the reserved observations catalog (ROC) after the completion of Spitzer's cryogenic mission to ensure that all archival data that can be used for this study are included in our sample. We found 1366 astronomical observation requests (AORs) containing high-resolution IRS spectra of extragalactic sources performed in either single-target or cluster mode.

We matched all IRS targets with optical spectroscopic catalogs of AGNs to identify type 1 sources with existing $M_{\mathrm{BH}}$ estimates and [O III] $5007 \AA$ detections that are required for the comparison of NLR kinematics at various wavelengths. For this purpose, we used (1) the reverberation mapping catalogs of Peterson et al. (2004), Bentz et al. (2006), Bentz et al. (2009), and Denney et al. (2010), (2) the spectroscopic subsample of the Palomar-Green (PG) QSOs (Boroson \& Green 1992; Vestergaard \& Peterson 2006), (3) the optical spectroscopic catalog of Marziani et al. (2003), (4) the Ho \& Kim (2009) catalog of type 1 AGNs, (5) the Sloan Digital Sky Survey (SDSS) Data Release 4 AGN catalogs of Kauffmann et al. (2003), of Greene \& Ho (2005), and of Netzer \& Trakhtenbrot (2007), and (6) the SDSS Data Release 7 (DR7). To identify AGNs in DR7, we used the Baldwin et al. (1981) diagnostic diagram of $[\mathrm{O}$ III $] / \mathrm{H}_{\beta}$ versus $\left[\mathrm{N}\right.$ II] $/ \mathrm{H}_{\alpha}$ with the $\mathrm{AGN}$ boundaries as updated by Kewley et al. (2006). We also examined sources that have a QSO target type, or a broad-line or an AGN spectral type subclass assigned by the SDSS pipeline. These samples also include a few type 2 AGNs, which we further complemented by matching all IRS targets with the Mulchaey et al. (1994), Turner et al. (1997), Bassani et al. (1999), Zakamska et al. (2003), Reyes et al. (2008), Bennert et al. (2009), and Liu et al. (2009) catalogs. A few additional optical spectra of type 2 AGNs were found in Spinelli et al. (2006).

In total, we found and reduced data sets from 370 Spitzer AORs, corresponding to high-resolution spectra of 298 sources. Resolved NLR lines were determined from the inspection of the [S IV] $10.51 \mu \mathrm{m}$, [Ne III] $15.56 \mu \mathrm{m}$, [O IV] $25.89 \mu \mathrm{m}$, or [Ne v] $14.32 \mu \mathrm{m}$ profiles as described in Section 3.2. To these 298 AGNs, we added six sources with fine-structure lines that were similarly resolved by $I S O$ short wavelength spectrograph (SWS) data (Sturm et al. 2002; Dasyra et al. 2008). The redshift distribution of all 304 sources in the final sample is shown in

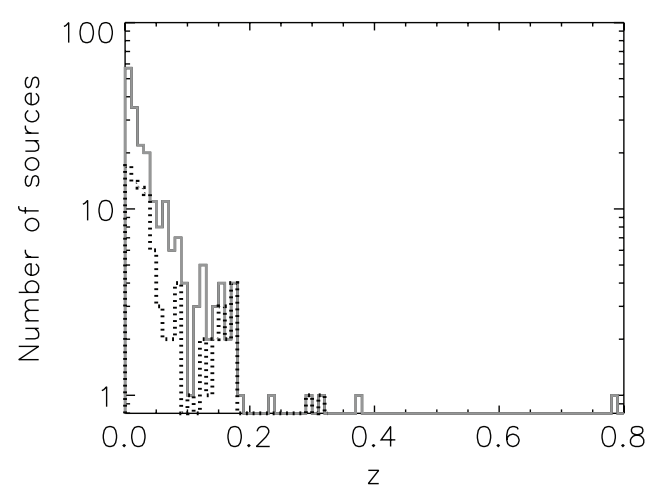

Figure 1. Redshift distribution of AGNs with MIR [S IV], [Ne III], [O IV], or $[\mathrm{NeV}]$ detections (gray solid line), and redshift distribution of AGNs with resolved [S IV], [Ne III], [O IV], or [Ne v] lines (black dotted line).

Figure 1. The 42 type 1 AGNs and the 39 type 2 AGNs that have resolved MIR narrow lines are presented in Tables 1 and 2, respectively.

\section{DATA REDUCTION \\ 3.1. Spectral Extraction}

We downloaded the Spitzer S15.0 pipeline basic calibration data (BCD) files. We used both staring and mapping observations, taken on either single-source or cluster mode.

The BCD files of the long-high (LH) wavelength data of each source were processed by the IDL routine DARK SETTLE, which is posted on the Spitzer Science Center (SSC) Web site, to correct for gradations of the dark current along the detector that leads to order tilting and mismatch. Using the short-high (SH) BCD files and the dark-settle corrected LH BCD files, we computed the average frame for each set of on-source observations, namely, for each target, nod position, and module. To identify cosmic ray hits, we also computed the median frame for the same set of observations and compared it to the average frame on a pixel-by-pixel basis. A pixel was flagged if the difference between the median and the average frame value differed by a factor of more than three times the rms noise in the median frame. For this pixel, the average value was replaced by the median value. For sources with available observations on sky, ${ }^{7}$ we also computed the median sky frame, which we subtracted from the on-source frame.

The next step was the removal of bad and rogue (i.e., slowly varying time response) pixels. We used the individual sky frames to create a generic bad pixel mask for all sources observed in a single Spitzer campaign, which we merged with the bad pixel map available at the SSC Web site for the same campaign. We then merged this generic mask with the mask of each individual (on-source and on-sky) exposure to create the mask for each science frame, nod position, and module. We proceeded to further masking of outliers, i.e., pixels that were located a couple of columns away from the edges of each spectral order, and that had a value exceeding the rms noise of the science frame. Their values were replaced with the median value of the frame, computed using only pixels in the useful detector range. A final visual inspection and a manual cleaning of the science frames

\footnotetext{
7 Several programs whose scientific goals required only the use of line fluxes (but not the use of equivalent widths or continuum flux measurements) did not acquire sky observations given that the flux of a line does not depend on its underlying continuum level.
} 
Table 1

Black Hole Masses and Optical NLR and Host Galaxy Properties of Type 1 AGNs

\begin{tabular}{|c|c|c|c|c|c|}
\hline Galaxy & $(2)$ & $\begin{array}{c}M_{\mathrm{BH}} \\
\left(10^{7} M_{\odot}\right) \\
(3)\end{array}$ & $\begin{array}{c}\log \left(L_{[\mathrm{O} \text { III }]} /\left(\mathrm{erg} \mathrm{s}^{-1}\right)\right) \\
(4)\end{array}$ & $\begin{array}{c}\sigma_{\left[\mathrm{O}_{\mathrm{III}}\right]}(5007 \AA) \\
\left(\mathrm{km} \mathrm{s}^{-1}\right) \\
(5)\end{array}$ & $\begin{array}{c}\sigma_{*} \\
\left(\mathrm{~km} \mathrm{~s}^{-1}\right) \\
(6)\end{array}$ \\
\hline 2MASSJ09184900+2117170 & 0.1490 & $3.45 \pm 0.35$ & 42.09 & 274 & $\ldots$ \\
\hline 2MASSiJ1659397+183436 & 0.1709 & $174.28 \pm 17.43$ & 42.55 & 249 & $183 \pm 10$ \\
\hline 2MASXJ10514428+3539304 & 0.1588 & $\ldots$ & 41.67 & 197 & $\ldots$ \\
\hline $3 \mathrm{C} 249.1$ & 0.3110 & $187.10 \pm 42.20$ & 43.03 & 292 & $\ldots$ \\
\hline $3 \mathrm{C} 273$ & 0.1583 & $88.60 \pm 18.70$ & 42.77 & 510 & $\ldots$ \\
\hline Ark120 & 0.0328 & $15.00 \pm 1.90$ & 41.34 & 209 & $\ldots$ \\
\hline CGCG121-075 & 0.0323 & $3.81 \pm 0.38$ & 41.56 & 238 & $\ldots$ \\
\hline ESO140-G043 & 0.0142 & $1.79 \pm 0.18$ & 41.09 & 89 & $\ldots$ \\
\hline FAIRALL9 & 0.0465 & $25.50 \pm 5.60$ & 42.08 & 181 & $\ldots$ \\
\hline IC4329A & 0.0160 & $9.47 \pm 0.95$ & 41.28 & 234 & $122 \pm 13$ \\
\hline IRAS13342+3932 & 0.1793 & $66.61 \pm 6.66$ & 42.30 & 234 & $104 \pm 14$ \\
\hline IRAS13451+1232 & 0.1217 & $135.96 \pm 13.60$ & 42.22 & 511 & $157 \pm 39$ \\
\hline IRAS18216+6418 & 0.2970 & $120.00 \pm 62.20$ & 43.93 & 302 & $\ldots$ \\
\hline MCG-2-58-22 & 0.0469 & $83.13 \pm 8.31$ & 42.18 & 208 & $\ldots$ \\
\hline Mrk 1014 & 0.1631 & $14.66 \pm 3.01$ & 42.96 & 324 & $200 \pm 60$ \\
\hline Mrk 279 & 0.0305 & $3.49 \pm 0.92$ & 41.47 & 247 & $197 \pm 12$ \\
\hline Mrk 335 & 0.0258 & $1.42 \pm 0.37$ & 41.52 & 119 & $\ldots$ \\
\hline Mrk 493 & 0.0313 & $0.63 \pm 0.06$ & 40.66 & 393 & $\ldots$ \\
\hline Mrk 590 & 0.0264 & $4.75 \pm 0.74$ & 41.24 & 182 & $192 \pm 10$ \\
\hline Mrk 704 & 0.0295 & $13.64 \pm 1.36$ & 41.40 & 140 & $\ldots$ \\
\hline Mrk 705 & 0.0290 & $39.63 \pm 9.18$ & 41.30 & 203 & $\ldots$ \\
\hline NGC 3227 & 0.0039 & $2.43 \pm 0.37$ & 40.33 & 206 & $134 \pm 6$ \\
\hline NGC 3516 & 0.0088 & $4.27 \pm 1.46$ & 40.91 & 106 & 235 \\
\hline NGC 4051 & 0.0023 & $0.17 \pm 0.05$ & 39.66 & 81 & $87 \pm 5$ \\
\hline NGC 4235 & 0.0080 & $\ldots$ & 39.53 & 170 & $143 \pm 34$ \\
\hline NGC 5548 & 0.0172 & $8.20 \pm 0.00$ & 41.44 & 210 & $192 \pm 15$ \\
\hline NGC 7469 & 0.0165 & $1.22 \pm 0.14$ & 41.54 & 153 & $142 \pm 3$ \\
\hline PG0026+129 & 0.1455 & $39.30 \pm 9.60$ & 42.46 & 191 & $\ldots$ \\
\hline PG0804+761 & 0.1010 & $69.30 \pm 8.30$ & 42.06 & 393 & $\ldots$ \\
\hline PG1119+120 & 0.0502 & $2.95 \pm 0.59$ & 41.49 & 249 & $162 \pm 28$ \\
\hline PG1229+204 & 0.0630 & $7.32 \pm 3.52$ & 41.68 & 197 & $162 \pm 32$ \\
\hline PG1351+640 & 0.0882 & $36.30 \pm 7.26$ & 42.19 & 390 & $\ldots$ \\
\hline PG1411+442 & 0.0896 & $44.30 \pm 14.60$ & 41.82 & 393 & $\ldots$ \\
\hline PG1426+015 & 0.0866 & $129.80 \pm 38.50$ & 41.89 & 287 & $217 \pm 15$ \\
\hline PG1440+356 & 0.0790 & $2.94 \pm 0.59$ & 41.48 & 393 & $\ldots$ \\
\hline PG1613+658 & 0.1295 & $27.90 \pm 12.90$ & 42.08 & 354 & $\ldots$ \\
\hline PG2130+099 & 0.0630 & $45.70 \pm 5.50$ & 41.93 & 296 & $172 \pm 46$ \\
\hline PG2349-014 & 0.1742 & $196.00 \pm 19.60$ & 43.11 & 221 & $223 \pm 36$ \\
\hline $3 \mathrm{C} 120^{\mathrm{a}}$ & 0.0330 & $5.55 \pm 2.70$ & 41.62 & 122 & $162 \pm 24$ \\
\hline Mrk $509^{\mathrm{a}}$ & 0.0344 & $14.30 \pm 1.20$ & 42.33 & 221 & $\ldots$ \\
\hline NGC $3783^{\mathrm{a}}$ & 0.0097 & $2.98 \pm 0.54$ & 41.43 & 98 & $95 \pm 10$ \\
\hline $\mathrm{NGC} 4151^{\mathrm{a}}$ & 0.0033 & $4.57 \pm 0.52$ & 41.44 & 181 & $95 \pm 7$ \\
\hline
\end{tabular}

Notes. Column 3: when possible, $M_{\mathrm{BH}}$ measurements are taken from reverberation mapping experiments (Peterson et al. 2004; Bentz et al. 2006; Bentz et al. 2009; Denney et al. 2010). Otherwise, they are taken from single-epoch spectroscopy (Vestergaard \& Peterson 2006; Kim et al. 2008) or computed from single-epoch spectroscopy (Marziani et al. 2003; Netzer \& Trakhtenbrot 2007; Ho \& Kim 2009; SDSS archival spectra) as in Vestergaard \& Peterson (2006). 2MASXJ10514428+3539304 has weak, broad $H_{\alpha}$ detection but no $H_{\beta}$ detection. Column 4: data from Whittle (1992a), Marziani et al. (2003), Ho \& Kim (2009), and SDSS DR7, as well as our own measurements from the spectra presented in Boroson \& Green (1992), performed as in Netzer \& Trakhtenbrot (2007). The luminosities are not corrected for extinction. Column 5: $\sigma_{[\mathrm{O} \text { III] }}$ values are taken from Whittle (1992a), Ho \& Kim (2009), or measured from SDSS spectra. Column 6: host galaxy velocity dispersion measurements are taken from Nelson et al. (2004), Onken et al. (2004), Dasyra et al. (2006a, 2006b, 2007), Ho (2007), and the seventh data release of SDSS. When more than one measurements were available, their average value was used. IRAS13451+1232 is a merger (Dasyra et al. 2006a). The velocity dispersion presented here corresponds to the combined value for both nuclei.

${ }^{\text {a }}$ Sources with MIR fine-structure lines resolved only by ISO.

were performed using the IRSCLEAN routine. All rogue pixels and outliers were flagged in the mask file.

The uncertainty of the average on-source frame was calculated as the square root of the sum of the squares of all individual uncertainty files, divided by the number of exposures. When sky observations were available, we computed the sky frame uncer- tainty in a similar way and combined it with the on-source frame uncertainty to produce the uncertainty of the final science frame.

We used the science frame, together with its uncertainty and mask files as inputs to the SSC software SPICE, which produces a one-dimensional spectrum from a two-dimensional spectral image. To extract the spectra, we used the regular extraction 
Table 2

Black Hole Masses and Optical NLR and Host Galaxy Properties of Type 2 AGNs

\begin{tabular}{|c|c|c|c|c|c|}
\hline Galaxy & (2) & $\begin{array}{c}M_{\mathrm{BH}} \\
\left(10^{7} M_{\odot}\right) \\
(3)\end{array}$ & $\begin{array}{c}\log \left(L_{[\mathrm{O} \text { III }]} /\left(\mathrm{erg} \mathrm{s}^{-1}\right)\right) \\
(4)\end{array}$ & $\begin{array}{c}\sigma_{\left[\mathrm{O}_{\text {III }}\right]}(5007 \AA) \\
\left(\mathrm{km} \mathrm{s}^{-1}\right) \\
(5)\end{array}$ & $\begin{array}{c}\sigma_{*} \\
\left(\mathrm{~km} \mathrm{~s}^{-1}\right) \\
(6)\end{array}$ \\
\hline 2MASXJ08035923+2345201 & 0.0297 & $\ldots$ & 40.99 & 121 & $130 \pm 5$ \\
\hline 2MASXJ08244333+2959238 & 0.0254 & $\ldots$ & 41.13 & 157 & $107 \pm 5$ \\
\hline 2MASXJ10181928+3722419 & 0.0497 & $\ldots$ & 41.33 & 155 & $100 \pm 6$ \\
\hline 2MASXJ12384342+0927362 & 0.0829 & $\ldots$ & 41.97 & 233 & $229 \pm 10$ \\
\hline 2MASXJ16164729+3716209 & 0.1518 & $\ldots$ & 42.51 & 150 & $229 \pm 9$ \\
\hline CGCG218-007 & 0.0273 & $\ldots$ & 40.85 & 131 & $149 \pm 5$ \\
\hline ESO103-G035 & 0.0133 & $\ldots$ & $\ldots$ & $\ldots$ & $\ldots$ \\
\hline IRAS05189-2524 & 0.0430 & $\ldots$ & 41.82 & 230 & $137 \pm 16$ \\
\hline IRAS15001+1433 & 0.1623 & $\ldots$ & 41.52 & 193 & $296 \pm 19$ \\
\hline IRAS18325-5926 & 0.0202 & $\ldots$ & $\ldots$ & $\ldots$ & $\ldots$ \\
\hline IRAS23060+0505 & 0.1730 & $\ldots$ & 42.24 & 243 & $\ldots$ \\
\hline MCG-03-34-064 & 0.0165 & $\ldots$ & $\ldots$ & $\ldots$ & $\ldots$ \\
\hline Mrk 1066 & 0.0120 & $\ldots$ & 40.88 & 172 & $\ldots$ \\
\hline Mrk 1457 & 0.0486 & $\ldots$ & 41.38 & 182 & $143 \pm 6$ \\
\hline Mrk 273 & 0.0378 & $\ldots$ & 40.79 & 255 & $244 \pm 18$ \\
\hline Mrk 3 & 0.0135 & $\ldots$ & 42.14 & 362 & $\ldots$ \\
\hline Mrk 463E & 0.0510 & $\ldots$ & 42.64 & 140 & $163 \pm 8$ \\
\hline Mrk 609 & 0.0345 & $\ldots$ & 41.32 & 215 & $145 \pm 4$ \\
\hline Mrk 622 & 0.0232 & $\ldots$ & 40.48 & 291 & $135 \pm 5$ \\
\hline NGC 1068 & 0.0038 & $0.86 \pm 0.03$ & 41.80 & 451 & $151 \pm 7$ \\
\hline NGC 1275 & 0.0174 & $\ldots$ & 41.68 & 601 & 246 \\
\hline NGC 2622 & 0.0286 & $\ldots$ & 41.43 & 255 & $\ldots$ \\
\hline NGC 2623 & 0.0185 & $\ldots$ & 39.01 & 140 & $152 \pm 9$ \\
\hline NGC $2639^{a}$ & 0.0111 & $\ldots$ & 39.70 & 226 & 188 \\
\hline NGC $3079^{a}$ & 0.0037 & $\ldots$ & 37.72 & 527 & 150 \\
\hline NGC 4258 & 0.0018 & $3.78 \pm 0.01$ & 40.87 & 174 & 167 \\
\hline NGC 4507 & 0.0118 & $\ldots$ & 41.53 & 91 & $\ldots$ \\
\hline NGC 5256 & 0.0279 & $\ldots$ & 41.91 & 176 & $187 \pm 8$ \\
\hline NGC 5506 & 0.0062 & $\ldots$ & 40.58 & 106 & $\ldots$ \\
\hline NGC 5728 & 0.0094 & $\ldots$ & 41.12 & 136 & $\ldots$ \\
\hline NGC 5929 & 0.0083 & $\ldots$ & 40.02 & 179 & $123 \pm 3$ \\
\hline NGC 6240 & 0.0245 & $\ldots$ & 39.02 & 426 & $229 \pm 43$ \\
\hline NGC 7172 & 0.0087 & $\ldots$ & $\ldots$ & $\ldots$ & $\ldots$ \\
\hline NGC 7674 & 0.0290 & $\ldots$ & 42.01 & 189 & $\ldots$ \\
\hline SBS1133+572 & 0.0516 & $\ldots$ & 41.47 & 168 & $207 \pm 7$ \\
\hline UGC 02608 & 0.0233 & $\ldots$ & $\ldots$ & $\ldots$ & $\ldots$ \\
\hline UGC 5101 & 0.0394 & $\ldots$ & 39.91 & 193 & $188 \pm 6$ \\
\hline Centaurus $\mathrm{A}^{\mathrm{b}}$ & 0.0018 & $4.90 \pm 1.40$ & $\ldots$ & $\ldots$ & $150 \pm 7$ \\
\hline Circinus $^{\mathrm{b}}$ & 0.0014 & $0.17 \pm 0.03$ & 38.55 & $\ldots$ & 80 \\
\hline
\end{tabular}

Notes. Column 3: $M_{\mathrm{BH}}$ measurements taken from Lodato \& Bertin (2003), Greenhill et al. (2003), Herrnstein et al. (2005), and Neumayer et al. (2007). When more than one measurements were available, we used the value with the lower uncertainty. Column 4: the luminosities are computed from Whittle (1992a), Oliva et al. (1994), Veilleux et al. (1995, 1999), Ho et al. (1997a), Marziani et al. (2003), or measured from SDSS spectra. They are not corrected for extinction. Column 5: $\sigma_{\text {[O III] }}$ values taken from Heckman et al. (1983), Whittle (1992a), Veilleux et al. (1999), or measured from SDSS spectra. Column 6: host galaxy velocity dispersions are compiled from Pahre (1999), Tecza et al. (2000), Tacconi et al. (2002), Dasyra et al. (2006b), Falcon-Barroso et al. (2006), Hinz \& Rieke (2006), Müller Sánchez et al. (2006), Ho (2007), Cappellari et al. (2009), Gültekin et al. (2009), and from the seventh data release of SDSS.

a Sources with shallow optical spectra, or spectra obtained under non-photometric conditions.

b Sources with MIR fine-structure lines resolved only by ISO.

mode, which equally weighs pixels when collapsing them along each row. We assumed a point-source extraction, since the LH and $\mathrm{SH}$ apertures $\left(22^{\prime \prime} .3 \times 11^{\prime \prime} .1\right.$ and $11^{\prime \prime} .3 \times 4$.". 7 , respectively $)$ are likely to include the bulk of the NLR emission for most sources in our sample. The end product of SPICE is the wavelength and flux calibrated spectrum for each individual order.

We merged the spectra of the various orders to a single spectrum for each nod position, clipping noisy edges (between 2 and 25 pixels, depending on the order). This task was performed for both the $\mathrm{SH}$ and the $\mathrm{LH}$ data sets. The $\mathrm{SH}$ and $\mathrm{LH}$ spectra were then merged to produce the full-range spectrum per nod position. The final spectrum of each object was produced by averaging the one-dimensional, full-range spectra of the two nod positions. Special care was taken not to merge sky-subtracted and non-sky-subtracted data sets for sources with observations from different programs. At the wavelengths of key atomic/ molecular lines, only nod positions without bad pixels were used, when possible. For all other pixels with a bad pixel flag 


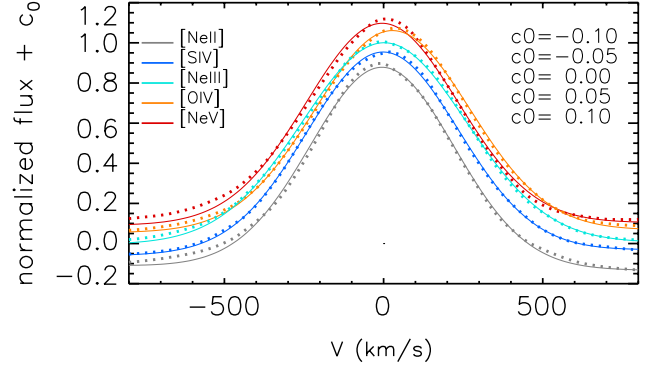

Figure 2. Stacked profiles (dotted lines) constructed using $29 z<0.02$ individual sources with unresolved line detections at all wavelengths. Solid lines correspond to the Gaussian best-fit solutions of the stacked profiles. The differences in the line peak positions are indicative of wavelength calibration uncertainties.

in one nod position, we only kept the value of the second nod position when the two values differed by more than the local rms noise value.

\subsection{Line Fitting}

As in Dasyra et al. (2008), ${ }^{8}$ we fitted all lines and their underlying continua using Gaussian and second-order polynomial functions, respectively. Gaussian fitting was preferred over direct FWHM computations which, given the resolution of IRS, can only be reliably performed for few high-velocity systems (e.g., Spoon \& Holt 2009). Gaussian fitting also suits best the studies of cloud kinematics on virial, regular, or symmetric motions, instead of clouds that are entrained by asymmetric outflows (Greene \& Ho 2005).

To claim a line detection, we required that its signal-to-noise ratio $(\mathrm{S} / \mathrm{N})$ was greater than 3 . To measure line widths, however,

8 All previously presented data were reanalyzed to account for changes in the Spitzer pipeline, and for enhancements in our bad-pixel identification routines. we only used lines with $\mathrm{S} / \mathrm{N}>5$ to avoid studying profiles of barely detected lines. We considered resolved all lines with $\sigma_{m}-\epsilon_{m}>\sigma_{i}+\epsilon_{i}$, where $\sigma_{m}$ is the measured velocity dispersion, $\sigma_{i}$ is the instrumental resolution at a given wavelength divided by 2.35 , and $\epsilon_{i}$ is the error of $\sigma_{i}$. The average resolution value in the $12.0-18.0 \mu \mathrm{m}$ range, which comprises all neon lines for the low $z$ galaxies, is $507 \pm 66 \mathrm{~km} \mathrm{~s}^{-1}$. The $25.0-34.2 \mu \mathrm{m}$ range, which comprises most of the $\mathrm{O}$ IV emission, has an average resolution of $503 \pm 63 \mathrm{~km} \mathrm{~s}^{-1}$. The error of $\sigma_{m}, \epsilon_{m}$, encapsulates both measurement and instrumental uncertainties. It was computed as $\left(\epsilon_{\mathrm{st}}^{2}+\epsilon_{i}^{2}\right)^{0.5}$, where $\epsilon_{\mathrm{st}}$ is the standard deviation of the different velocity dispersion values that were obtained for each line when using different polynomial functions to describe its underlying continuum. Intrinsic velocity dispersions, $\sigma$, were computed as $\left(\sigma_{m}^{2}-\sigma_{i}^{2}\right)^{0.5}$, converted to rest frame, and presented in Tables 1 and 2 for key fine-structure lines in all sources.

To assess possible systematic errors in the wavelength calibration, we normalized and stacked the line profiles of 29 sources with detected but unresolved emission lines of [ $\left.\mathrm{Ne}_{\mathrm{II}}\right]$, [S IV], [Ne III], [O IV], and [Ne v] (Figure 2). The stacking of unresolved lines in different $z$ sources permitted for a finer sampling of the instrumental resolution curve than that determined by the detector pixel size. We only used sources at $z<0.02$ to study systematic effects within a few pixels from the rest-frame wavelength of each line. This $z$ cutoff translates to 25 pixels at the center of the $\mathrm{SH}$ array range, $14.7 \mu \mathrm{m}$. We found that line peak offsets due to such systematics are typically limited to $50 \mathrm{~km} \mathrm{~s}^{-1}$.

\section{RESULTS}

\subsection{Identifying NLR Tracers in the MIR}

Of the 304 AGNs in our sample, 300 had spectral coverage at $10.51 \mu \mathrm{m}$, and 143 showed [S IV] emission. Similarly, 226 sources had spectral coverage at $25.89 \mu \mathrm{m}$, and 135 had [O IV] detections. Thus, the typical line detection rate was of the order of $\sim 50 \%$.

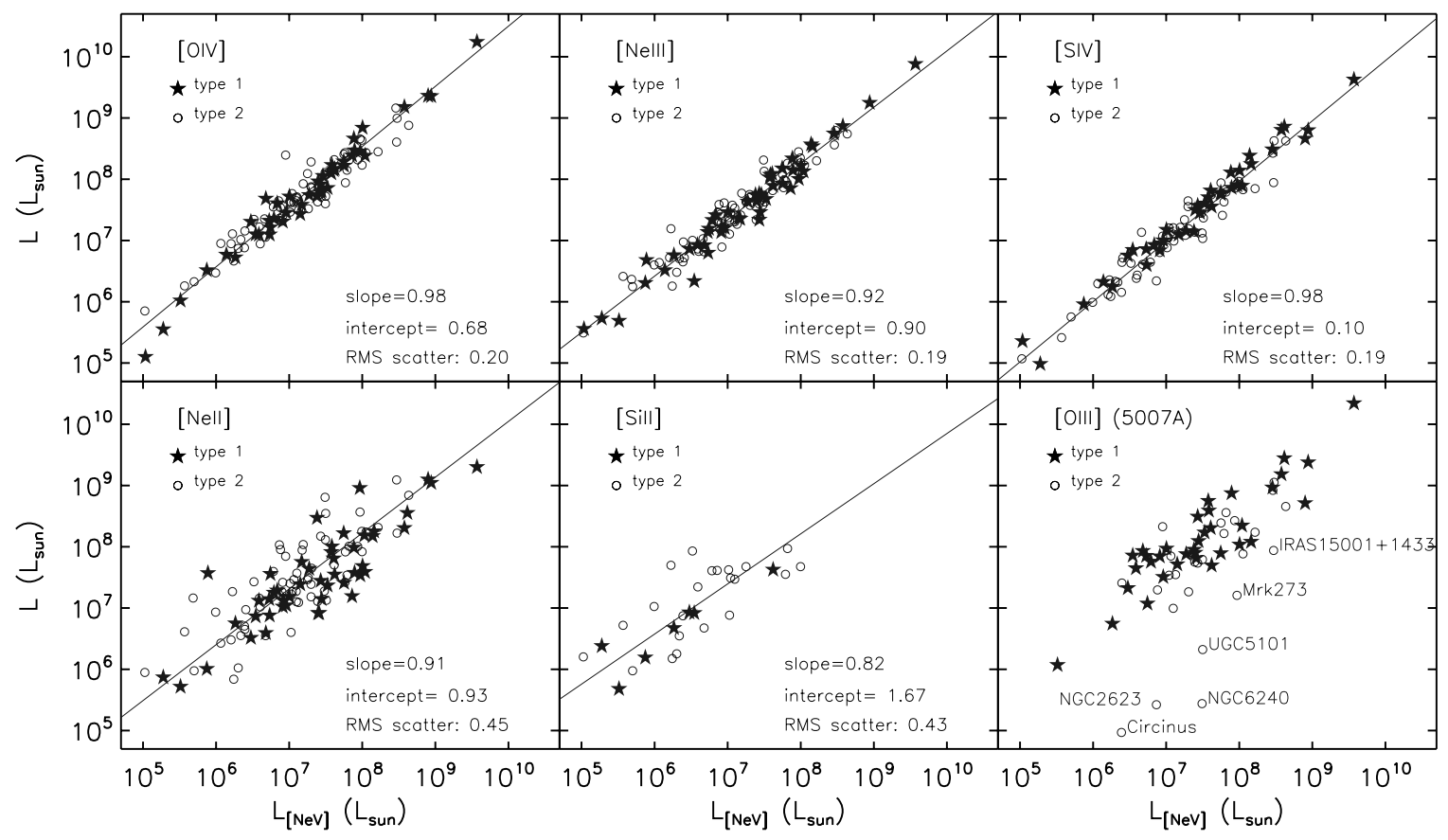

Figure 3. Relation of the [Ne v] luminosity, commonly used as an AGN indicator, with the luminosities of other fine-structure lines in MIR and optical wavelengths. The optical luminosities are not corrected for extinction. 

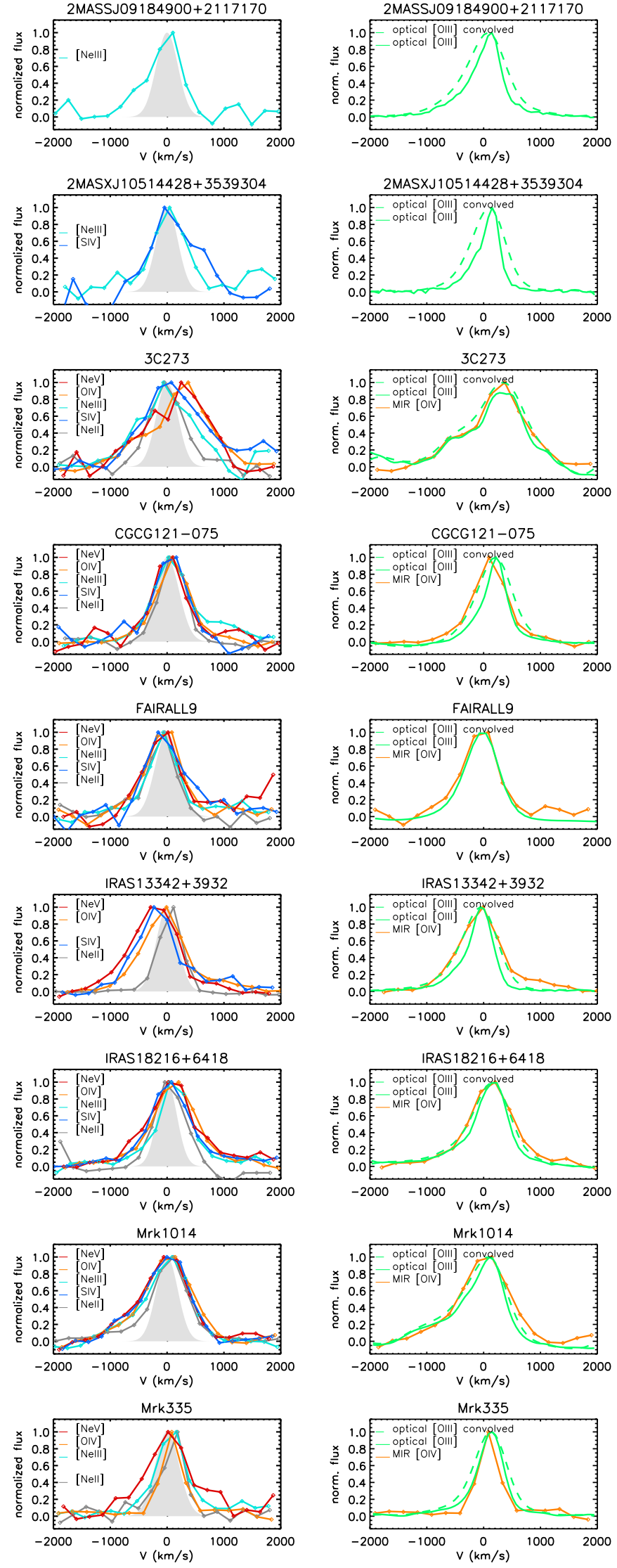
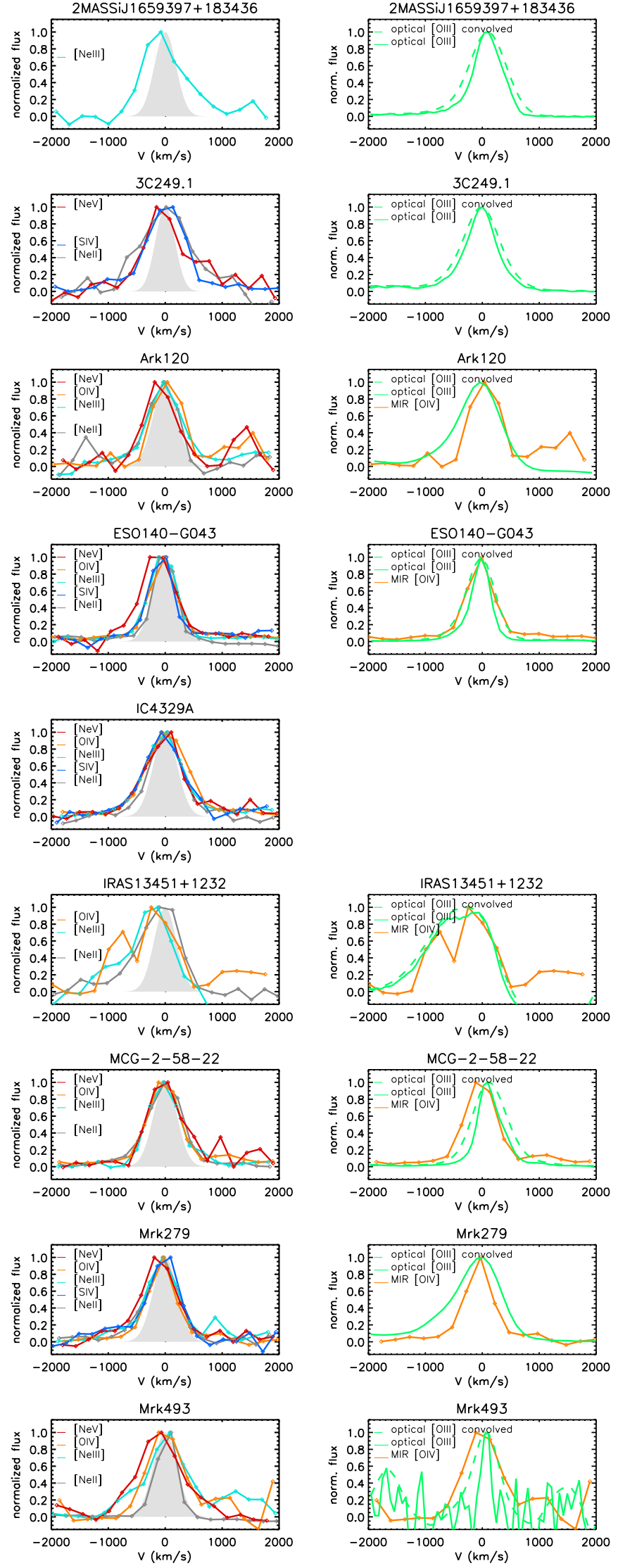

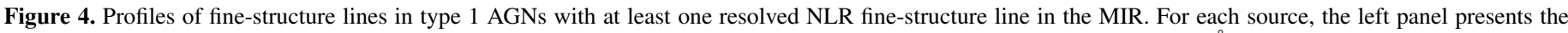

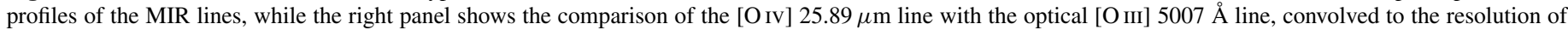
the IRS data. The filled area corresponds to the average IRS resolution at the wavelengths of those lines. 

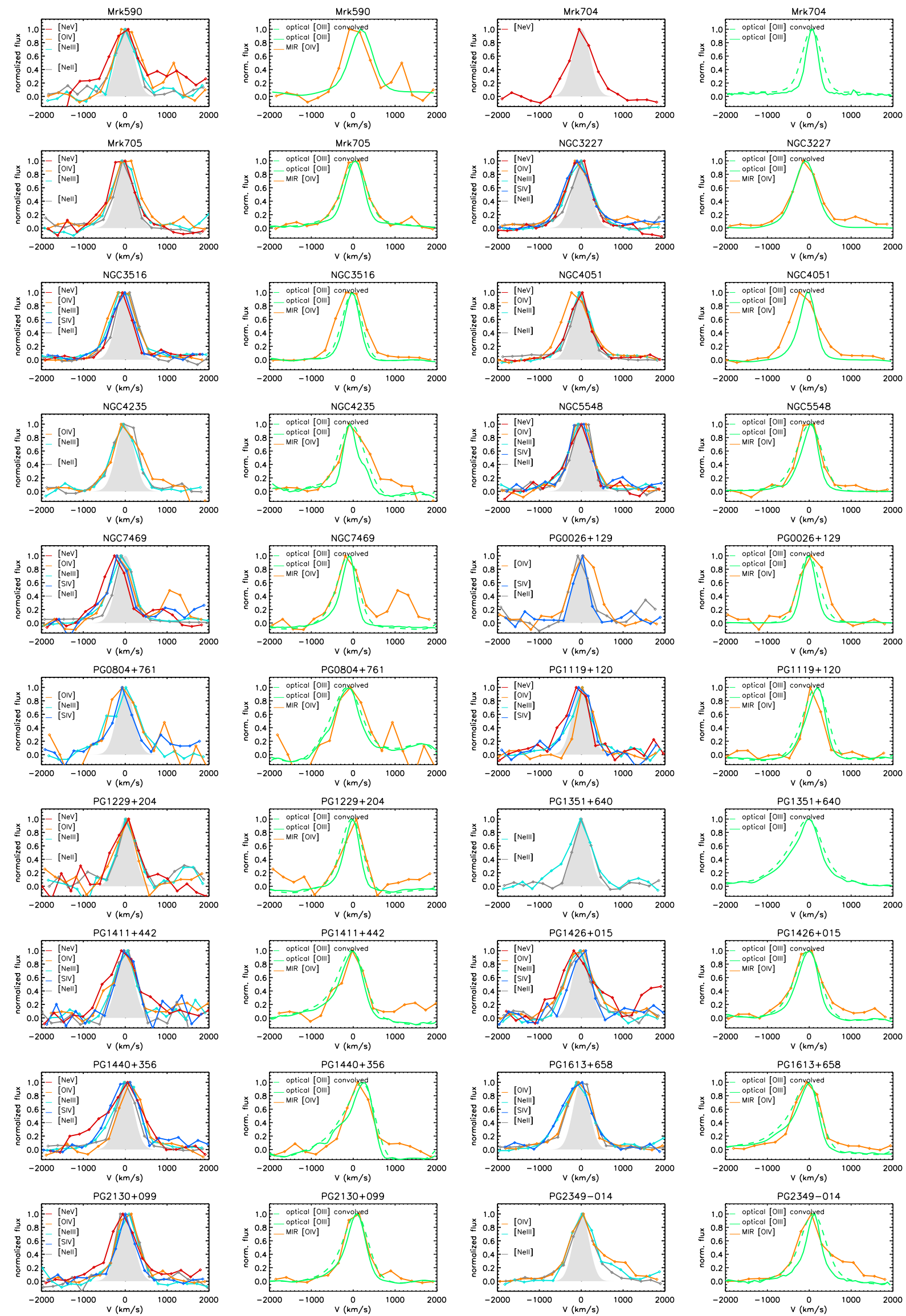

Figure 4. (Continued) 

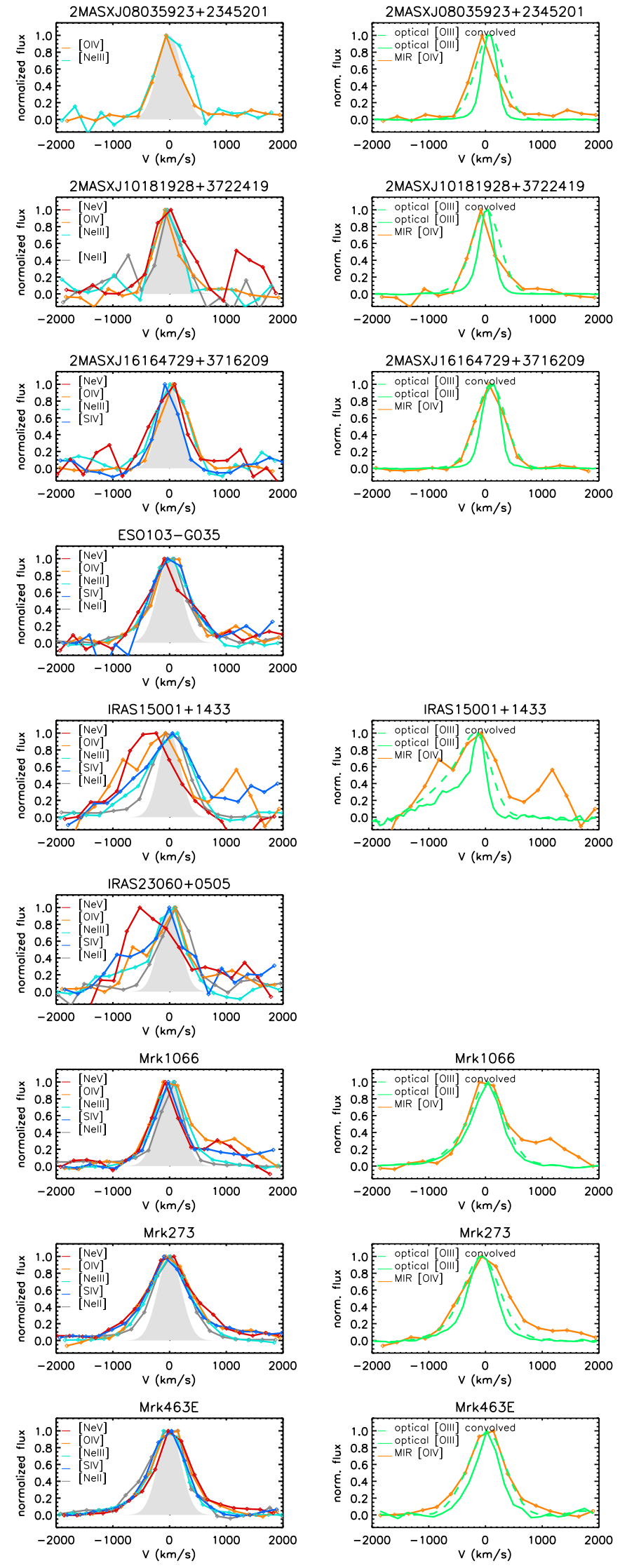

Figure 5. Profiles of fine-structure lines in type 2 AGNs with at least one NLR line resolved in the MIR.
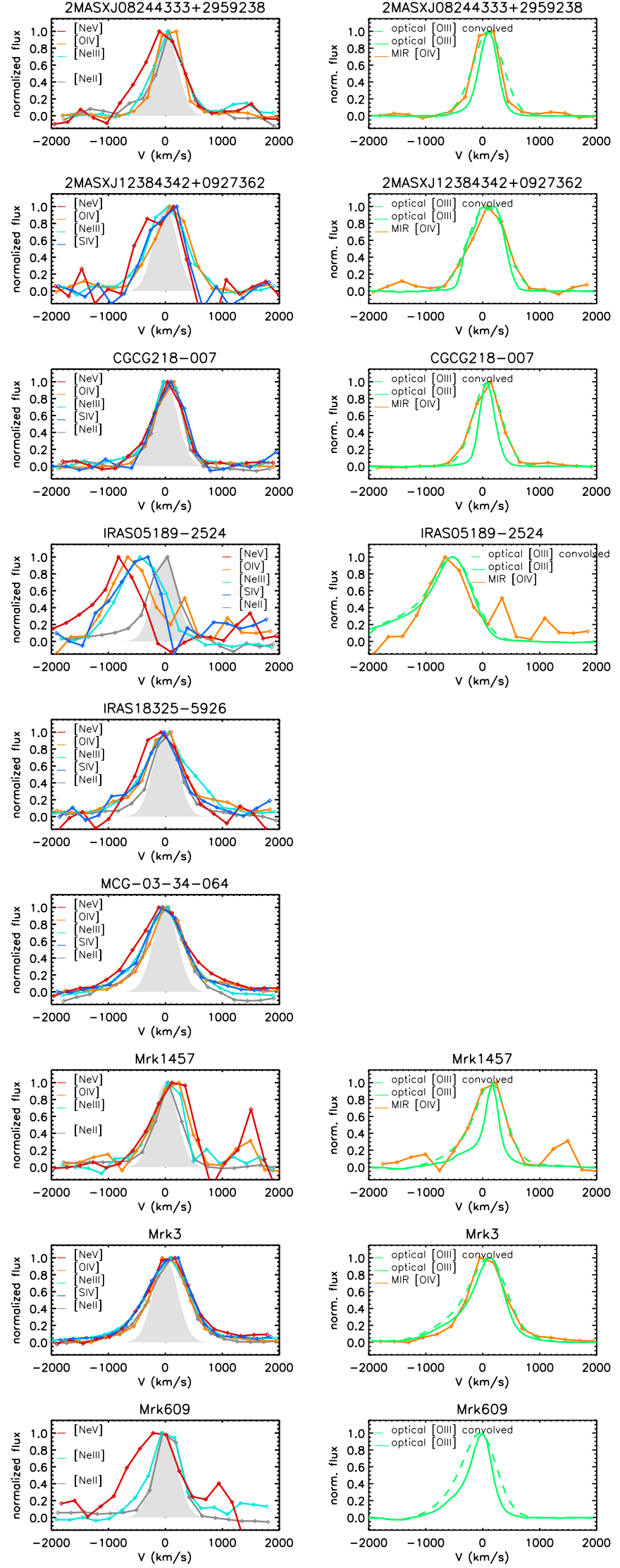

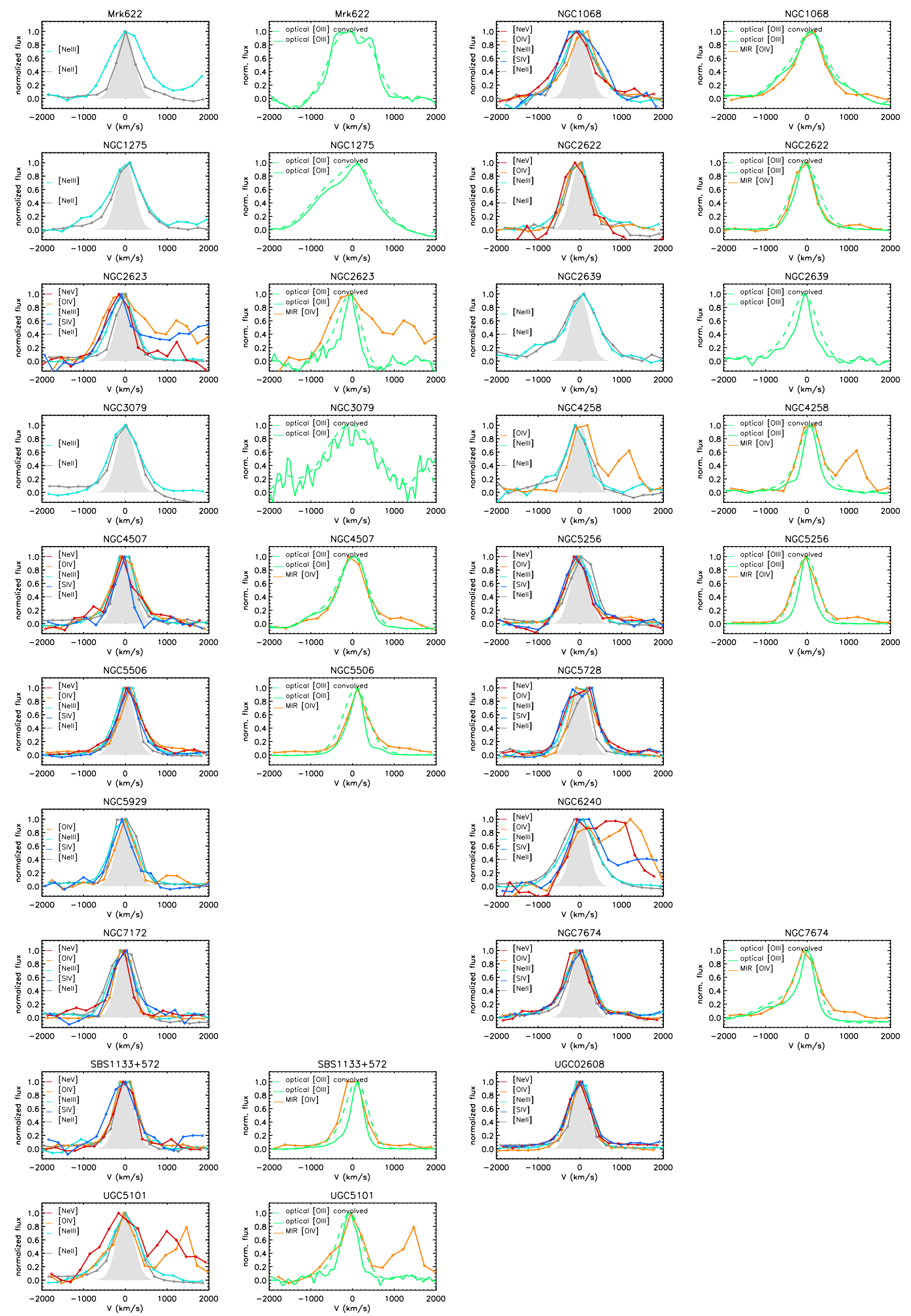

Figure 5. (Continued) 
Table 3

Fluxes and Velocity Dispersions of Ionized Gas Narrow Lines in Type 1 AGNs

\begin{tabular}{|c|c|c|c|c|c|c|c|c|c|c|}
\hline Galaxy & $\begin{array}{c}f_{[\mathrm{Ne} \mathrm{II]}]} \\
\left(10^{-18} \mathrm{~W} \mathrm{~m}^{-2}\right)\end{array}$ & $\begin{array}{c}f_{[\mathrm{S} \mathrm{IV}]} \\
\left(10^{-18} \mathrm{~W} \mathrm{~m}^{-2}\right)\end{array}$ & $\begin{array}{c}f_{[\mathrm{Ne} \mathrm{III]}]} \\
\left(10^{-18} \mathrm{~W} \mathrm{~m}^{-2}\right)\end{array}$ & $\begin{array}{c}f_{\left[\mathrm{O}_{\text {Iv }}\right]} \\
\left(10^{-18} \mathrm{~W} \mathrm{~m}^{-2}\right)\end{array}$ & $\begin{array}{c}f_{[\mathrm{Ne} \mathrm{v}]} \\
\left(10^{-18} \mathrm{~W} \mathrm{~m}^{-2}\right)\end{array}$ & $\begin{array}{c}\sigma_{[\mathrm{Ne} \mathrm{II]}} \\
\left(\mathrm{km} \mathrm{s}^{-1}\right)\end{array}$ & $\begin{array}{c}\sigma_{[\mathrm{S} \mathrm{IV}]} \\
\left(\mathrm{km} \mathrm{s}^{-1}\right)\end{array}$ & $\begin{array}{c}\sigma_{[\mathrm{Ne} \mathrm{III]}} \\
\left(\mathrm{km} \mathrm{s}^{-1}\right)\end{array}$ & $\begin{array}{c}\sigma_{[\mathrm{O} \text { IV] }} \\
\left(\mathrm{km} \mathrm{s}^{-1}\right)\end{array}$ & $\begin{array}{c}\sigma_{[\mathrm{Ne} \mathrm{v}]} \\
\left(\mathrm{km} \mathrm{s}^{-1}\right)\end{array}$ \\
\hline 2MASSJ09184900+2117170 & $<3.61$ & $<10.86$ & $15.65 \pm 2.39$ & $\ldots$ & $<25.07$ & $\ldots$ & $\ldots$ & $206 \pm 35$ & $\ldots$ & $\ldots$ \\
\hline 2MASSiJ1659397+183436 & $<10.67$ & $<10.16$ & $26.79 \pm 2.95$ & $\ldots$ & $<10.15$ & $\ldots$ & $\ldots$ & $277 \pm 52$ & $\ldots$ & $\ldots$ \\
\hline 2MASXJ10514428+3539304 & $<6.21$ & $9.97 \pm 1.98$ & $19.45 \pm 2.11$ & $\ldots$ & $<5.20$ & $\ldots$ & $325 \pm 48$ & $\ldots$ & $\ldots$ & $\ldots$ \\
\hline $3 \mathrm{C} 249.1$ & $4.37 \pm 0.40$ & $8.85 \pm 0.43$ & & & $5.05 \pm 0.59$ & $370 \pm 65$ & $286 \pm 33$ & & & $329 \pm 36$ \\
\hline $3 \mathrm{C} 273$ & $11.42 \pm 1.67$ & $36.04 \pm 3.33$ & $41.58 \pm 2.61$ & $85.45 \pm 6.42$ & $21.20 \pm 4.21$ & $230 \pm 39$ & $470 \pm 87$ & $402 \pm 47$ & $450 \pm 67$ & $495 \pm 57$ \\
\hline Ark120 & $28.97 \pm 2.53$ & $<13.56$ & $35.23 \pm 2.71$ & $36.84 \pm 3.70$ & $10.04 \pm 1.61$ & $202 \pm 32$ & & $213 \pm 38$ & $190 \pm 39$ & \\
\hline CGCG121-075 & $24.88 \pm 1.44$ & $24.39 \pm 2.00$ & $47.28 \pm 1.96$ & $85.25 \pm 1.86$ & $16.36 \pm 1.25$ & $\ldots$ & $209 \pm 50$ & $238 \pm 42$ & $220 \pm 27$ & $223 \pm 43$ \\
\hline ESO140-G043 & $96.32 \pm 2.92$ & $87.41 \pm 2.95$ & $139.5 \pm 2.2$ & $247.6 \pm 4.9$ & $79.10 \pm 2.19$ & $\ldots$ & $\ldots$ & $\ldots$ & $\ldots$ & $203 \pm 29$ \\
\hline FAIRALL9 & $21.50 \pm 1.85$ & $28.59 \pm 3.86$ & $44.80 \pm 3.44$ & $63.73 \pm 3.68$ & $20.79 \pm 3.77$ & $\ldots$ & $296 \pm 45$ & $197 \pm 36$ & $258 \pm 48$ & $231 \pm 42$ \\
\hline IC4329A & $241.5 \pm 6.2$ & $245.7 \pm 9.5$ & $535.0 \pm 6.8$ & $1009 \pm 14$ & $286.1 \pm 8.4$ & $227 \pm 32$ & $235 \pm 29$ & $256 \pm 30$ & $275 \pm 31$ & $282 \pm 34$ \\
\hline IRAS13342+3932 & $53.68 \pm 1.11$ & $19.74 \pm 1.27$ & $<28.42$ & $98.54 \pm 1.72$ & $33.98 \pm 0.98$ & $\ldots$ & $310 \pm 48$ & $\ldots$ & $324 \pm 29$ & $403 \pm 38$ \\
\hline IRAS13451+1232 & $45.66 \pm 1.82$ & $<5.59$ & $<70.31$ & $27.87 \pm 5.24$ & $<7.86$ & $311 \pm 37$ & $\ldots$ & $\ldots$ & $479 \pm 54$ & $\ldots$ \\
\hline IRAS18216+6418 & $27.52 \pm 2.06$ & $58.26 \pm 1.23$ & $104.5 \pm 3.5$ & $240.1 \pm 12.3$ & $50.79 \pm 1.69$ & $214 \pm 34$ & $304 \pm 31$ & $237 \pm 31$ & $343 \pm 36$ & $390 \pm 34$ \\
\hline MCG-2-58-22 & $74.71 \pm 1.86$ & $<33.31$ & $87.08 \pm 3.79$ & $126.3 \pm 3.6$ & $28.26 \pm 2.63$ & $\ldots$ & $\ldots$ & $183 \pm 33$ & $187 \pm 32$ & $204 \pm 43$ \\
\hline Mrk 1014 & $58.26 \pm 1.42$ & $33.20 \pm 1.67$ & $93.20 \pm 2.96$ & $119.9 \pm 5.8$ & $46.01 \pm 2.39$ & $253 \pm 37$ & $376 \pm 36$ & $379 \pm 41$ & $379 \pm 39$ & $380 \pm 48$ \\
\hline Mrk 279 & $81.42 \pm 2.88$ & $26.48 \pm 2.04$ & $80.35 \pm 3.97$ & $102.8 \pm 3.6$ & $34.80 \pm 2.19$ & $215 \pm 34$ & $237 \pm 36$ & $222 \pm 35$ & $\ldots$ & $248 \pm 42$ \\
\hline Mrk 335 & $10.54 \pm 0.90$ & $<25.80$ & $22.56 \pm 1.75$ & $130.0 \pm 3.7$ & $12.88 \pm 1.86$ & $\ldots$ & $\ldots$ & $\ldots$ & $155 \pm 17$ & $247 \pm 64$ \\
\hline Mrk 493 & $62.69 \pm 1.31$ & $<23.47$ & $27.09 \pm 1.53$ & $28.42 \pm 4.76$ & $9.59 \pm 1.27$ & $\ldots$ & $\ldots$ & $280 \pm 39$ & $267 \pm 46$ & $280 \pm 38$ \\
\hline Mrk 590 & $32.81 \pm 2.12$ & $<16.58$ & $21.14 \pm 2.54$ & $31.04 \pm 3.06$ & $9.57 \pm 1.64$ & $\ldots$ & $\ldots$ & $197 \pm 33$ & $200 \pm 33$ & $206 \pm 40$ \\
\hline Mrk 704 & $<10.05$ & $<61.44$ & $<50.82$ & $<151.67$ & $51.08 \pm 3.04$ & $\ldots$ & $\ldots$ & $\ldots$ & $\ldots$ & $249 \pm 37$ \\
\hline Mrk 705 & $52.30 \pm 1.90$ & $<23.26$ & $49.59 \pm 2.11$ & $57.82 \pm 2.19$ & $30.18 \pm 1.66$ & $\ldots$ & $\ldots$ & $192 \pm 31$ & $210 \pm 35$ & $280 \pm 39$ \\
\hline NGC 3227 & $708.7 \pm 25.2$ & $220.9 \pm 5.5$ & $725.8 \pm 6.2$ & $668.0 \pm 17.6$ & $231.2 \pm 7.5$ & $\ldots$ & $241 \pm 32$ & $228 \pm 30$ & $216 \pm 29$ & $209 \pm 34$ \\
\hline NGC 3516 & $74.01 \pm 2.29$ & $128.9 \pm 4.4$ & $164.4 \pm 2.9$ & $458.4 \pm 5.5$ & $67.57 \pm 2.18$ & $\ldots$ & $\ldots$ & $198 \pm 30$ & $229 \pm 28$ & $\ldots$ \\
\hline NGC 4051 & $172.6 \pm 4.9$ & $<47.59$ & $162.0 \pm 2.9$ & $347.6 \pm 7.1$ & $107.7 \pm 3.9$ & $\ldots$ & $\ldots$ & $172 \pm 29$ & $295 \pm 27$ & $\ldots$ \\
\hline NGC 4235 & $34.85 \pm 1.62$ & $<6.03$ & $33.29 \pm 1.77$ & $33.60 \pm 3.04$ & $<5.28$ & $\ldots$ & $\ldots$ & $214 \pm 34$ & $286 \pm 43$ & $\ldots$ \\
\hline NGC 5548 & $83.08 \pm 3.32$ & $42.90 \pm 2.55$ & $81.76 \pm 2.77$ & $124.7 \pm 8.6$ & $31.50 \pm 2.16$ & $\ldots$ & $\ldots$ & $177 \pm 30$ & $\ldots$ & $206 \pm 38$ \\
\hline NGC 7469 & $1915 \pm 27$ & $90.00 \pm 7.88$ & $357.9 \pm 7.5$ & $340.0 \pm 38.0$ & $154.4 \pm 10.0$ & $\ldots$ & $158 \pm 12$ & $196 \pm 29$ & $196 \pm 45$ & $240 \pm 44$ \\
\hline PG0026+129 & $2.56 \pm 0.33$ & $4.94 \pm 0.43$ & $<7.72$ & $20.16 \pm 4.01$ & $<5.07$ & $\ldots$ & $\ldots$ & & $305 \pm 40$ & $\ldots$ \\
\hline PG0804+761 & $<4.44$ & $15.53 \pm 2.28$ & $20.89 \pm 1.70$ & $20.74 \pm 2.87$ & $<11.13$ & $\ldots$ & $292 \pm 111$ & $323 \pm 47$ & $342 \pm 66$ & $\ldots$ \\
\hline PG1119+120 & $<4.00$ & $20.63 \pm 2.55$ & $28.41 \pm 2.01$ & $60.00 \pm 2.37$ & $16.16 \pm 2.33$ & $\ldots$ & $\ldots$ & $\ldots$ & $\ldots$ & $193 \pm 47$ \\
\hline PG1229+204 & $5.84 \pm 0.59$ & $<20.90$ & $12.05 \pm 1.37$ & $26.74 \pm 3.55$ & $11.48 \pm 1.13$ & $\ldots$ & $\ldots$ & $\ldots$ & $\ldots$ & $218 \pm 50$ \\
\hline PG1351+640 & $21.92 \pm 0.99$ & $<10.26$ & $29.39 \pm 1.73$ & $<12.38$ & $<3.83$ & $\ldots$ & $\ldots$ & $200 \pm 43$ & $\ldots$ & $\ldots$ \\
\hline PG1411+442 & $4.54 \pm 0.79$ & $7.79 \pm 1.00$ & $9.24 \pm 0.62$ & $13.99 \pm 2.31$ & $6.49 \pm 0.58$ & $\ldots$ & $\ldots$ & $\ldots$ & $212 \pm 45$ & $316 \pm 63$ \\
\hline PG1426+015 & $13.46 \pm 1.08$ & $13.67 \pm 2.28$ & $25.55 \pm 1.09$ & $29.51 \pm 4.38$ & $8.47 \pm 1.09$ & $226 \pm 38$ & $\ldots$ & $258 \pm 36$ & $292 \pm 57$ & $289 \pm 57$ \\
\hline PG1440+356 & $42.72 \pm 0.96$ & $15.55 \pm 1.70$ & $38.57 \pm 1.26$ & $50.22 \pm 2.37$ & $14.26 \pm 0.86$ & $193 \pm 34$ & $211 \pm 39$ & $253 \pm 35$ & $253 \pm 38$ & $304 \pm 107$ \\
\hline PG1613+658 & $37.66 \pm 1.23$ & $13.25 \pm 1.01$ & $30.90 \pm 1.02$ & $66.49 \pm 3.15$ & $<7.92$ & $226 \pm 30$ & $269 \pm 33$ & $289 \pm 33$ & $225 \pm 29$ & $\ldots$ \\
\hline PG2130+099 & $16.14 \pm 1.22$ & $32.54 \pm 1.53$ & $55.73 \pm 2.88$ & $100.8 \pm 3.7$ & $45.16 \pm 3.01$ & $\ldots$ & $\ldots$ & $187 \pm 30$ & $\ldots$ & $287 \pm 45$ \\
\hline PG2349-014 & $15.95 \pm 0.71$ & $<6.36$ & $20.51 \pm 1.01$ & $34.73 \pm 3.82$ & $<6.83$ & $273 \pm 48$ & $\ldots$ & $259 \pm 34$ & $308 \pm 35$ & $\ldots$ \\
\hline $3 \mathrm{C} 120$ & $78.86 \pm 3.72$ & $225.7 \pm 3.3$ & $271.6 \pm 3.7$ & $1129 \pm 7$ & $163.8 \pm 2.7$ & $\ldots$ & $\ldots$ & $\ldots$ & $114 \pm 8$ & $111 \pm 16$ \\
\hline Mrk 509 & $118.0 \pm 4.1$ & $73.19 \pm 3.92$ & $153.0 \pm 3.4$ & $180.0 \pm 5.1$ & $54.04 \pm 2.63$ & $173 \pm 35$ & $\ldots$ & $\ldots$ & $165 \pm 30$ & \\
\hline NGC 3783 & $197.0 \pm 3.3$ & $128.8 \pm 5.1$ & $255.9 \pm 5.0$ & $380.0 \pm 8.5$ & $152.0 \pm 5.2$ & $\ldots$ & $\ldots$ & $244 \pm 12$ & $141 \pm 24$ & $133 \pm 21$ \\
\hline NGC 4151 & $1180 \pm 12$ & $1130 \pm 22$ & $350.0 \pm 13.9$ & $2030 \pm 33$ & $560.0 \pm 11.0$ & $\ldots$ & $207 \pm 16$ & $155 \pm 7$ & $169 \pm 12$ & $139 \pm 25$ \\
\hline
\end{tabular}

Notes. No data for the line fluxes indicate no spectral coverage at the observed-frame wavelengths of these lines. No data for the velocity dispersion of detected lines indicate that their signal-to-noise ratio was between 3 and 5 , or that their profiles were unresolved. 
Table 4

Fluxes and Velocity Dispersions of Ionized Gas Narrow Lines in Type 2 AGNs

\begin{tabular}{|c|c|c|c|c|c|c|c|c|c|c|}
\hline Galaxy & $\begin{array}{c}f_{[\mathrm{Ne} \mathrm{II]}} \\
\left(10^{-18} \mathrm{~W} \mathrm{~m}^{-2}\right)\end{array}$ & $\begin{array}{c}f_{[\mathrm{S} \text { IV }]} \\
\left(10^{-18} \mathrm{~W} \mathrm{~m}^{-2}\right)\end{array}$ & $\begin{array}{c}f_{[\mathrm{Ne} \mathrm{III]}]} \\
\left(10^{-18} \mathrm{~W} \mathrm{~m}^{-2}\right)\end{array}$ & $\begin{array}{c}f_{[\mathrm{O} \text { IV }]} \\
\left(10^{-18} \mathrm{~W} \mathrm{~m}^{-2}\right)\end{array}$ & $\begin{array}{c}f_{[\mathrm{Ne} \mathrm{v}]} \\
\left(10^{-18} \mathrm{~W} \mathrm{~m}^{-2}\right)\end{array}$ & $\begin{array}{c}\sigma_{[\mathrm{Ne} \mathrm{II}]} \\
\left(\mathrm{km} \mathrm{s}^{-1}\right)\end{array}$ & $\begin{array}{c}\sigma_{[\mathrm{S} \mathrm{IV}]} \\
\left(\mathrm{km} \mathrm{s}^{-1}\right)\end{array}$ & $\begin{array}{c}\sigma_{[\mathrm{Ne} \mathrm{III]}} \\
\left(\mathrm{km} \mathrm{s}^{-1}\right)\end{array}$ & $\begin{array}{c}\sigma_{[\mathrm{O} \text { IV }]} \\
\left(\mathrm{km} \mathrm{s}^{-1}\right)\end{array}$ & $\begin{array}{c}\sigma_{[\mathrm{Ne} \mathrm{v}]} \\
\left(\mathrm{km} \mathrm{s}^{-1}\right)\end{array}$ \\
\hline 2MASXJ08035923+2345201 & $<6.39$ & $<5.50$ & $18.78 \pm 2.08$ & $28.45 \pm 1.59$ & $<4.75$ & $\ldots$ & $\ldots$ & $203 \pm 42$ & $\ldots$ & $\ldots$ \\
\hline 2MASXJ08244333+2959238 & $33.17 \pm 2.04$ & $<20.88$ & $49.47 \pm 3.44$ & $83.64 \pm 2.13$ & $36.14 \pm 2.78$ & $207 \pm 39$ & $\ldots$ & $\ldots$ & $\ldots$ & $264 \pm 30$ \\
\hline 2MASXJ10181928+3722419 & $8.67 \pm 1.53$ & $<6.35$ & $14.69 \pm 1.87$ & $28.81 \pm 1.60$ & $16.59 \pm 2.80$ & $\ldots$ & $\ldots$ & $\ldots$ & $\ldots$ & $200 \pm 32$ \\
\hline 2MASXJ12384342+0927362 & $<8.33$ & $20.09 \pm 2.25$ & $27.85 \pm 2.15$ & $58.83 \pm 2.38$ & $12.94 \pm 2.35$ & $\ldots$ & $232 \pm 42$ & $260 \pm 42$ & $261 \pm 36$ & $265 \pm 73$ \\
\hline 2MASXJ16164729+3716209 & $<5.34$ & $16.53 \pm 1.49$ & $22.55 \pm 2.52$ & $90.14 \pm 2.28$ & $17.88 \pm 2.23$ & $\ldots$ & $\ldots$ & $\ldots$ & $\ldots$ & $211 \pm 37$ \\
\hline CGCG218-007 & $102.6 \pm 3.1$ & $35.72 \pm 2.64$ & $86.07 \pm 2.69$ & $166.7 \pm 2.3$ & $46.59 \pm 1.98$ & $\ldots$ & $\ldots$ & $\ldots$ & $\ldots$ & $215 \pm 33$ \\
\hline ESO103-G035 & $294.0 \pm 7.6$ & $115.1 \pm 10.1$ & $414.0 \pm 9.4$ & $311.7 \pm 13.9$ & $172.9 \pm 10.5$ & $223 \pm 37$ & $232 \pm 34$ & $276 \pm 31$ & $240 \pm 35$ & $346 \pm 52$ \\
\hline IRAS05189-2524 & $191.8 \pm 8.9$ & $65.30 \pm 6.26$ & $186.1 \pm 7.1$ & $261.2 \pm 37.2$ & $152.6 \pm 12.0$ & $253 \pm 42$ & $335 \pm 55$ & $392 \pm 41$ & $444 \pm 63$ & $404 \pm 43$ \\
\hline IRAS15001+1433 & $66.48 \pm 1.36$ & $4.74 \pm 0.82$ & $27.97 \pm 0.69$ & $21.78 \pm 3.21$ & $15.89 \pm 1.97$ & $247 \pm 34$ & $388 \pm 36$ & $348 \pm 38$ & $558 \pm 96$ & $422 \pm 81$ \\
\hline IRAS18325-5926 & $367.9 \pm 7.8$ & $114.9 \pm 8.2$ & $436.0 \pm 5.3$ & $390.7 \pm 12.2$ & $262.6 \pm 9.6$ & $181 \pm 31$ & $265 \pm 39$ & $367 \pm 32$ & $300 \pm 34$ & $404 \pm 64$ \\
\hline IRAS23060+0505 & $32.26 \pm 2.40$ & $19.65 \pm 2.34$ & $25.61 \pm 2.10$ & $35.31 \pm 5.45$ & $20.12 \pm 2.69$ & $278 \pm 59$ & $366 \pm 127$ & $285 \pm 62$ & $343 \pm 57$ & $419 \pm 62$ \\
\hline MCG-03-34-064 & $514.9 \pm 8.2$ & $476.1 \pm 8.3$ & $1150 \pm 13$ & $1053 \pm 22$ & $615.6 \pm 8.2$ & $344 \pm 34$ & $298 \pm 33$ & $361 \pm 28$ & $273 \pm 30$ & $435 \pm 36$ \\
\hline Mrk 1066 & $1094 \pm 21$ & $102.0 \pm 7.4$ & $469.1 \pm 7.6$ & $418.1 \pm 22.1$ & $92.38 \pm 8.31$ & $\ldots$ & $\ldots$ & $216 \pm 32$ & $284 \pm 41$ & $246 \pm 48$ \\
\hline Mrk 1457 & $91.99 \pm 2.39$ & $<14.09$ & $38.13 \pm 4.09$ & $28.10 \pm 2.54$ & $22.09 \pm 3.04$ & $\ldots$ & $\ldots$ & $\ldots$ & $228 \pm 43$ & $310 \pm 60$ \\
\hline Mrk 273 & $444.9 \pm 7.9$ & $101.7 \pm 2.3$ & $338.1 \pm 2.5$ & $550.1 \pm 17.8$ & $112.3 \pm 3.7$ & $252 \pm 30$ & $357 \pm 34$ & $332 \pm 30$ & $357 \pm 38$ & $406 \pm 32$ \\
\hline Mrk 3 & $979.8 \pm 10.2$ & $592.7 \pm 6.2$ & $1749 \pm 11$ & $1964 \pm 24$ & $632.5 \pm 7.5$ & $276 \pm 29$ & $322 \pm 29$ & $314 \pm 29$ & $263 \pm 28$ & $327 \pm 31$ \\
\hline Mrk 463E & $108.2 \pm 3.5$ & $275.0 \pm 5.4$ & $404.6 \pm 7.3$ & $641.8 \pm 11.3$ & $193.9 \pm 4.7$ & $308 \pm 35$ & $284 \pm 30$ & $235 \pm 29$ & $295 \pm 29$ & $287 \pm 31$ \\
\hline Mrk 609 & $213.5 \pm 6.2$ & $<22.18$ & $56.71 \pm 2.53$ & $82.43 \pm 25.69$ & $38.69 \pm 4.28$ & $\ldots$ & $\ldots$ & $202 \pm 36$ & $\ldots$ & $488 \pm 60$ \\
\hline Mrk 622 & $97.29 \pm 13.80$ & $<8.61$ & $49.29 \pm 2.82$ & $<37.42$ & $<38.06$ & $\ldots$ & $\ldots$ & $333 \pm 34$ & $\ldots$ & \\
\hline NGC 1068 & $4988 \pm 183$ & $6199 \pm 336$ & $13781 \pm 246$ & $20406 \pm 473$ & $8974 \pm 221$ & $359 \pm 52$ & $414 \pm 44$ & $383 \pm 31$ & $364 \pm 48$ & $363 \pm 39$ \\
\hline NGC 1275 & $461.5 \pm 8.0$ & $<16.26$ & $223.7 \pm 5.6$ & $<84.37$ & $<11.65$ & $299 \pm 31$ & $\ldots$ & $260 \pm 31$ & $\ldots$ & $\ldots$ \\
\hline NGC 2622 & $62.02 \pm 2.63$ & $<18.46$ & $80.41 \pm 2.93$ & $89.29 \pm 3.58$ & $24.73 \pm 2.08$ & $\ldots$ & $\ldots$ & $213 \pm 33$ & $\ldots$ & $289 \pm 39$ \\
\hline NGC 2623 & $550.1 \pm 9.7$ & $11.20 \pm 1.36$ & $147.2 \pm 1.7$ & $117.4 \pm 18.2$ & $37.55 \pm 2.97$ & $\ldots$ & $\ldots$ & $213 \pm 31$ & $357 \pm 52$ & $222 \pm 39$ \\
\hline NGC 2639 & $86.23 \pm 2.66$ & $<6.90$ & $48.88 \pm 1.95$ & $<23.82$ & $<7.51$ & $346 \pm 43$ & $\ldots$ & $336 \pm 49$ & $\ldots$ & $\ldots$ \\
\hline NGC 3079 & $1310 \pm 60$ & $<14.61$ & $238.3 \pm 3.3$ & $<94.19$ & $<38.79$ & $198 \pm 33$ & $\ldots$ & $285 \pm 29$ & & $\ldots$ \\
\hline NGC 4258 & $123.5 \pm 7.3$ & $<15.61$ & $70.61 \pm 5.61$ & $79.40 \pm 8.03$ & $<13.67$ & $193 \pm 42$ & $\ldots$ & $191 \pm 36$ & $204 \pm 53$ & $\ldots$ \\
\hline NGC 4507 & $307.8 \pm 6.9$ & $93.08 \pm 6.84$ & $287.8 \pm 6.3$ & $354.1 \pm 11.6$ & $125.7 \pm 6.4$ & $\ldots$ & $\ldots$ & $220 \pm 35$ & $318 \pm 42$ & $345 \pm 81$ \\
\hline NGC 5256 & $160.6 \pm 3.1$ & $27.06 \pm 1.56$ & $94.40 \pm 1.45$ & $569.8 \pm 10.3$ & $20.54 \pm 1.37$ & $227 \pm 29$ & $239 \pm 37$ & $227 \pm 30$ & $191 \pm 28$ & $202 \pm 40$ \\
\hline NGC 5506 & $850.6 \pm 14.3$ & $735.4 \pm 15.6$ & $1537 \pm 11$ & $2262 \pm 40$ & $568.0 \pm 10.6$ & $\ldots$ & $239 \pm 33$ & $207 \pm 28$ & $\ldots$ & $208 \pm 32$ \\
\hline NGC 5728 & $366.2 \pm 7.7$ & $317.2 \pm 8.2$ & $536.5 \pm 6.7$ & $1155 \pm 12$ & $217.1 \pm 3.4$ & $\ldots$ & $272 \pm 41$ & $263 \pm 29$ & $306 \pm 30$ & $314 \pm 32$ \\
\hline NGC 5929 & $104.8 \pm 2.2$ & $12.41 \pm 1.98$ & $95.07 \pm 1.40$ & $43.67 \pm 6.18$ & $<6.09$ & $\ldots$ & & $210 \pm 28$ & $\ldots$ & $\ldots$ \\
\hline NGC 6240 & $1872 \pm 29$ & $38.7 \pm 2.9$ & $599.9 \pm 4.2$ & $374.0 \pm 35.9$ & $90.24 \pm 9.02$ & $362 \pm 37$ & $279 \pm 52$ & $341 \pm 29$ & $\ldots$ & $\ldots$ \\
\hline NGC 7172 & $334.6 \pm 8.1$ & $55.74 \pm 4.43$ & $168.1 \pm 4.0$ & $384.4 \pm 5.3$ & $90.23 \pm 3.49$ & $256 \pm 30$ & $\ldots$ & $204 \pm 30$ & $\ldots$ & $\ldots$ \\
\hline NGC 7674 & $215.7 \pm 5.2$ & $152.6 \pm 5.9$ & $346.0 \pm 6.5$ & $442.9 \pm 12.1$ & $184.4 \pm 7.0$ & $\ldots$ & $267 \pm 34$ & $253 \pm 32$ & $242 \pm 32$ & $267 \pm 39$ \\
\hline SBS1133+572 & $108.3 \pm 2.9$ & $42.01 \pm 3.62$ & $105.3 \pm 3.8$ & $168.7 \pm 2.8$ & $69.89 \pm 2.87$ & $\ldots$ & $248 \pm 40$ & $\ldots$ & $\ldots$ & $\ldots$ \\
\hline UGC 02608 & $566.5 \pm 9.4$ & $292.8 \pm 9.5$ & $706.3 \pm 6.1$ & $1378 \pm 17$ & $312.9 \pm 5.1$ & $\ldots$ & $213 \pm 31$ & & & \\
\hline UGC 5101 & $379.7 \pm 9.0$ & $<7.34$ & $146.2 \pm 2.1$ & $57.18 \pm 9.66$ & $34.19 \pm 3.73$ & $254 \pm 40$ & $\ldots$ & $350 \pm 34$ & $285 \pm 41$ & $385 \pm 40$ \\
\hline Centaurus A & $2210 \pm 45$ & $140.0 \pm 9.6$ & $1400 \pm 12$ & $980.0 \pm 42.2$ & $200.0 \pm 8.5$ & $220 \pm 31$ & & $134 \pm 21$ & $127 \pm 9$ & $132 \pm 21$ \\
\hline Circinus & $4536 \pm 145$ & $1270 \pm 58$ & $4000 \pm 90$ & $6793 \pm 420$ & $2180 \pm 101$ & $\ldots$ & $91 \pm 6$ & $86 \pm 4$ & $113 \pm 3$ & $90 \pm 8$ \\
\hline
\end{tabular}

Note. Same as in Table 3. 

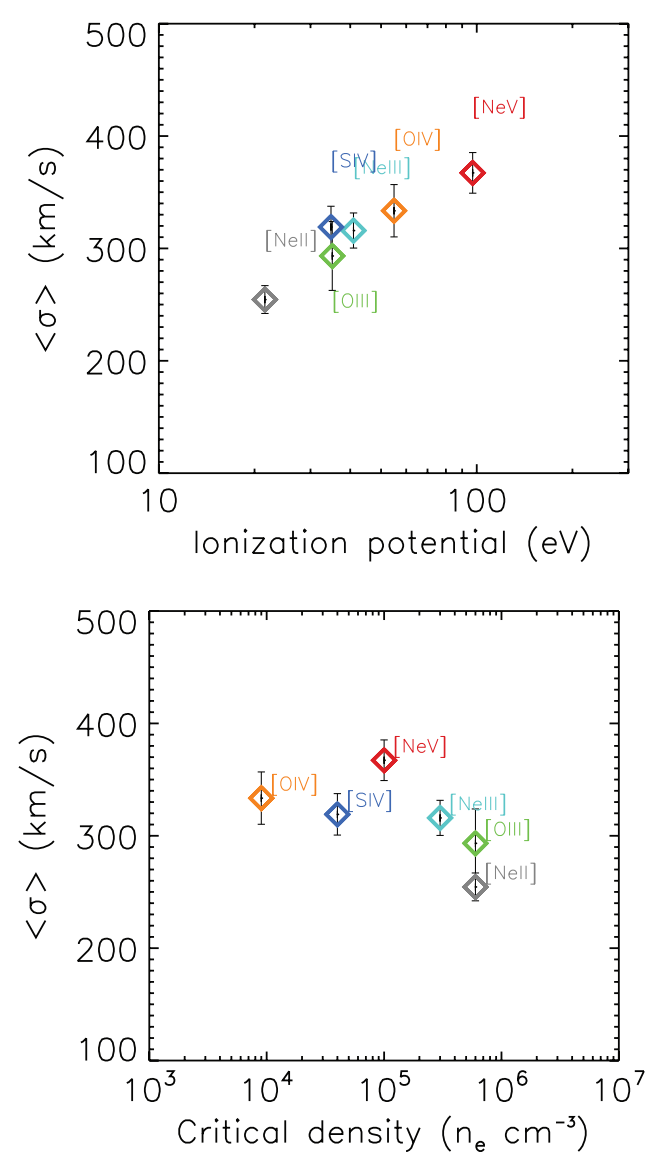

Figure 6. Average NLR gas velocity dispersion (corrected for instrumental broadening) as a function of ionization potential (upper panel) and critical density in units of electrons $n_{e}$ per cubic centimeter (lower panel) for 16 sources with detections in all of the lines used for the construction of this figure.

We plotted the luminosities of the most frequently observed MIR lines against that of [ $\mathrm{Ne} \mathrm{v}]$ to identify fine-structure lines that can be reliably used as tracers of the gas that is photoionized by the AGN (Figure 3). Given that photons of at least $97.12 \mathrm{eV}$ are required to ionize $\mathrm{Ne}$ IV to $\mathrm{Ne} \mathrm{V}$, its ionization source must be far-ultraviolet or soft X-ray radiation from the AGN. We find that the [Ne v] luminosity is on a tight correlation of 0.2 dex scatter with the [O IV] and [S IV] luminosities (Figure 3; PereiraSantaella et al. 2010), and we further confirm that the [Ne v] and [Ne III] luminosities trace each other well (Gorjian et al. 2007). Therefore, the Ne v, O IV, Ne III, and S IV ions are most likely to be primarily excited by the same mechanism. However, the scatter between the luminosities of [Ne V] and [ $\mathrm{Ne} \mathrm{II]} \mathrm{or} \mathrm{[Si} \mathrm{II]}$ is approximately twice as high, of 0.4-0.5 dex. Ne II and Si II have low ionization potentials ( 21.56 and $8.15 \mathrm{eV}$, respectively), thus, a non-negligible part of their line emission could be tracing star-forming regions in the AGN host galaxy.

The excellent, almost one-to-one correlation over several orders of magnitude in luminosity that is seen between the [Ne v] $14.32 \mu \mathrm{m}$ and [S IV] $10.51 \mu \mathrm{m}$ lines does not include any extinction correction. This result indicates that the bulk of the [S IV] emission is not strongly affected by the silicate absorption feature centered at $9.7 \mu \mathrm{m}$, which is seen in several of our targets with moderate or high optical depths, i.e., $\tau>0.5$. Thus, the geometric distribution of the silicates is likely to be more compact than the size of the NLR (see also Soifer et al. 2002; Tristram et al. 2007; Schweitzer et al. 2008). While obscuration effects do not significantly affect the relative fluxes

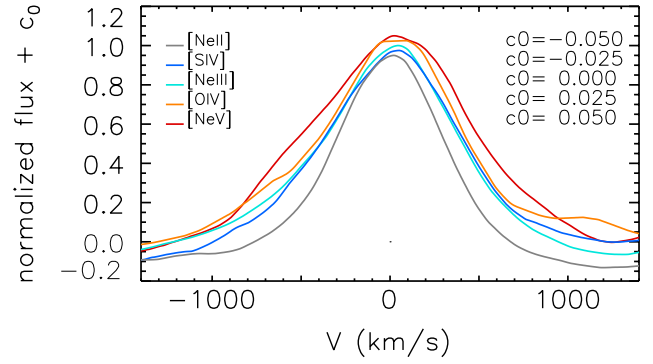

Figure 7. Stacked MIR line profiles of the 16 sources used in Figure 6.

of MIR lines, the [O III] $5007 \AA$ emission can suffer from strong extinction primarily in type 2 AGNs (Figure 3 ).

\subsection{Line Profiles: Velocity Dispersion of Different Ionized Gas Components}

Our query for sources with resolved [S IV], [Ne III], [O IV], or [Ne v] lines resulted in 81 AGNs with kinematic information of the NLR gas in the MIR (Figures 4 and 5). The different fine-structure lines that were used as primary tracers of clouds photoionized by the AGN often led to different $\sigma$ measurements (Figures 4 and 5). Differences in the ionization potential of the various ionic species can play a major role in determining the observed line profiles, alongside with differences in the critical density for collisional de-excitation of the various transitions, and with light extinction by dust particles. We find the [Nev] line to be often broader than lines from ions of lower ionization potential (e.g., Mrk 590; Figures 4 and 5; Tables 3 and 4). A significant increase in the average velocity dispersion with increasing ionization potential is shown in Figures 6 and 7, as found for 16 sources with resolved profiles in all [Ne II], [O III], [S IV], [Ne III], [O IV], and [Ne v] lines. This result provides evidence for a stratification of the NLR clouds, i.e., for ions of high ionization being located nearer to the $\mathrm{BH}$ than ions of low ionization potential (Filippenko \& Halpern 1984; Oliva et al. 1994; Ho 2009).

Radially dependent line widths will also be produced if the NLR has a gas density gradient increasing with proximity to the BH (Filippenko \& Halpern 1984; Ferguson et al. 1997), which is not connected to a mean ionization level gradient. The line widths will be affected as the line fluxes from transitions of low critical density for collisional de-excitation, $n_{\mathrm{c}}$, will be preferentially suppressed at dense environments. We observe no trend of $\sigma$ with $n_{\mathrm{c}}$ (Figure 6), suggesting that the typical NLR gas densities are below $n_{\mathrm{c}}$ for the MIR transitions that we examined. The lack of dependence of the average $\sigma$ on $n_{\mathrm{c}}$ demonstrates the power of MIR lines in probing the NLR kinematics. One counter example to this statistical finding could be MCG-03-34-064. Because all of its neon lines have higher velocity dispersions than [O IV] and [S IV], its NLR density could be of the order of $10^{4}$ hydrogen atoms per cubic centimeter.

Dust either mixed or outside the NLR gas clouds can lead to different extinction of lines at different $\lambda$ (Groves et al. 2004; Winter et al. 2010). Because MIR lines are less affected by obscuration than optical lines, they could be probing clouds that are nearer to the $\mathrm{BH}$, where obscuration is often higher. In this case, their widths could be broader than those of optical lines, in particular in type 2 AGNs. Mrk 273, NGC 2623, and IRAS $15001+1433$ do have larger [O IV] than [O III] widths and low $[\mathrm{O} \mathrm{III}] /[\mathrm{O} \mathrm{IV}]$ flux ratios (see Figure 3 ), but their [O III] and $[\mathrm{S}$ IV] or $[\mathrm{Ne} \mathrm{III}]$ widths are in good agreement with each 


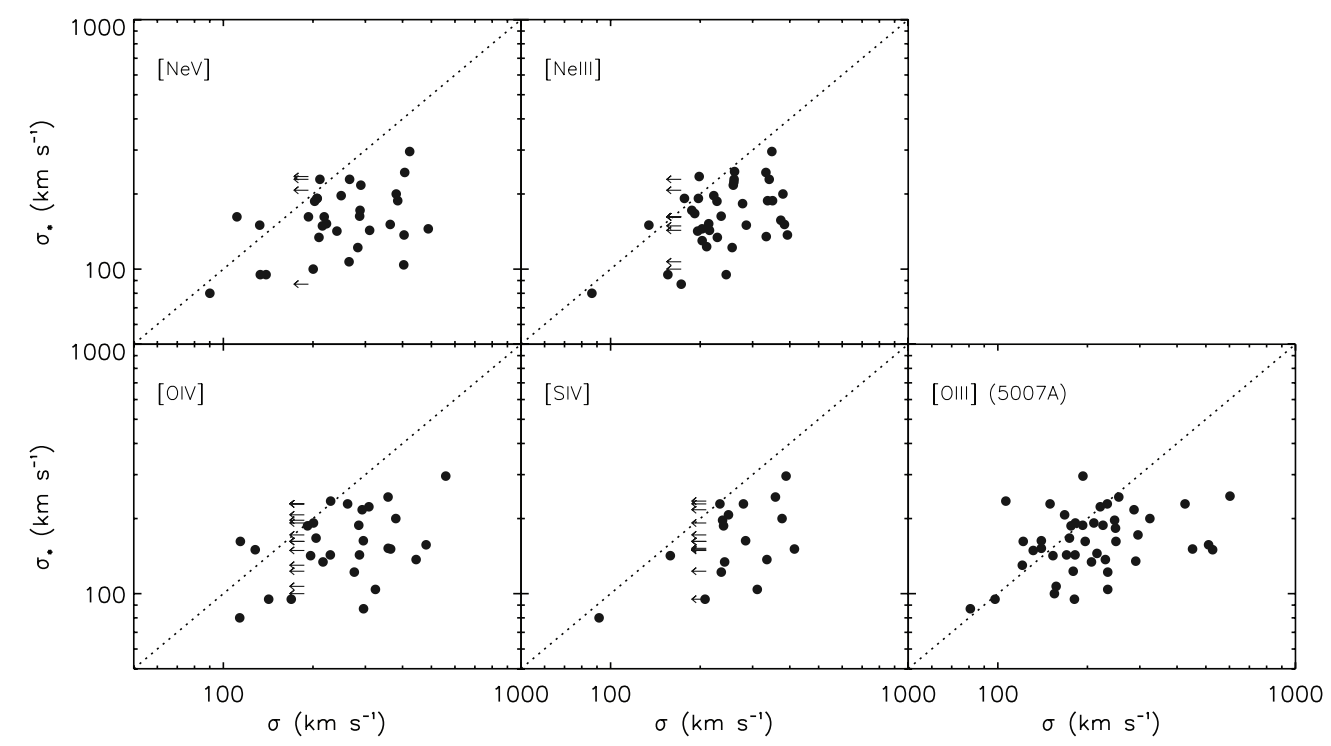

Figure 8. Comparison of the stellar velocity dispersion with the NLR gas velocity dispersion, as measured from MIR lines and from the optical [O III] $5007 \AA$ line.

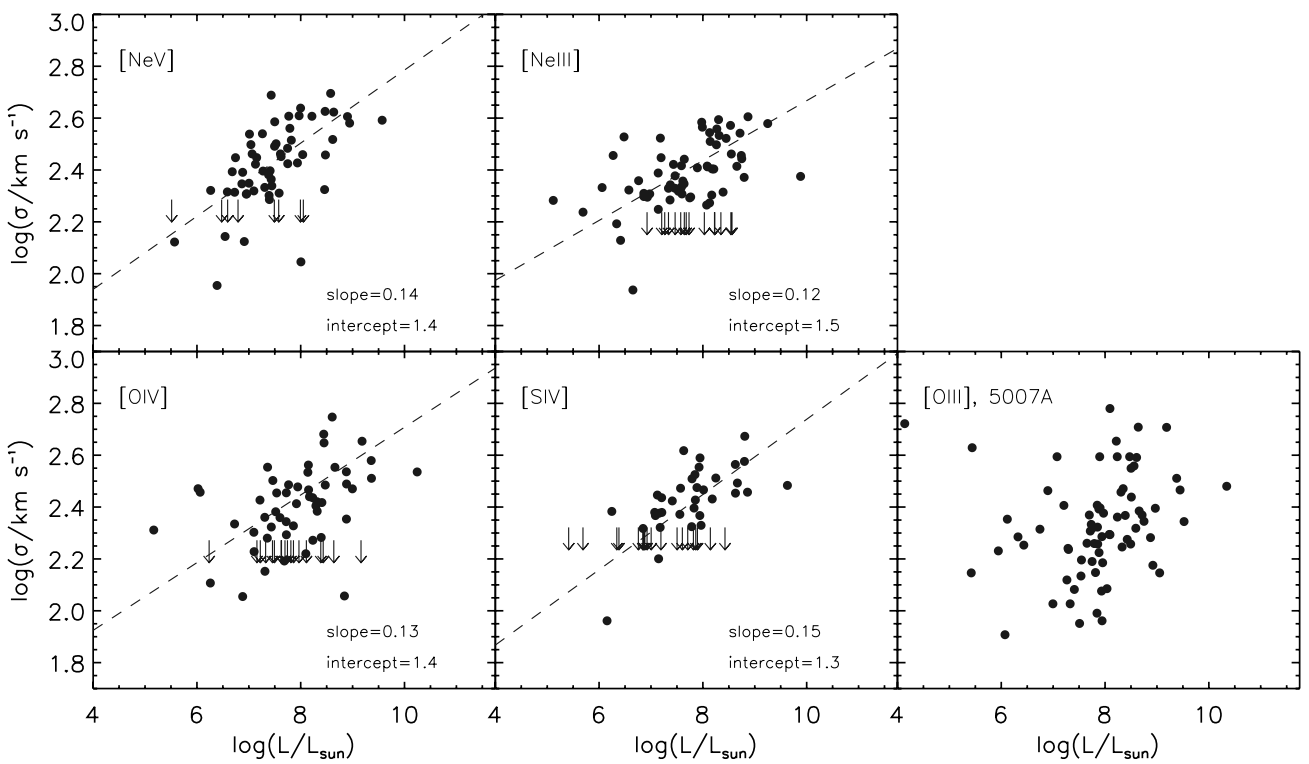

Figure 9. Velocity dispersion increase with the luminosity of the line from which it was measured. The error-weighed best-fit solution for each MIR line is presented with a dashed line.

other. The comparable ionization potentials of O III, S IV, and Ne III, i.e., 35.12, 34.79, and $40.96 \mathrm{eV}$, respectively, ascribe again any profile differences to ionization effects. We conclude that obscuration is not a common driver of differences in the profiles of optical and IR lines. The nuclear obscuration could be high enough to hide blue wings, associated with outflows moving away from the observer, even in IR wavelengths.

A simple comparison of the NLR gas velocity dispersion to that of the stars in the host galaxy shows that $\sigma$ is not identical to $\sigma_{*}$ (Figure 8 ). The gas velocity dispersion systematically exceeds that of the stars, with a large scatter between the two quantities. The average velocity dispersion excess is in the range $50-100 \mathrm{~km} \mathrm{~s}^{-1}$ for all lines. The excess could be intrinsically higher, given that the optical observations had a slit width that was often an order of magnitude narrower than the IRS slit width. The origin of this excess could be related to either gas clouds that are accelerated by AGN-feedback mechanisms (Ho 2009) or to virialized gas clouds with a more compact spatial distribution than that of the stars when probed by emission lines from ions of ionization potential $\gtrsim 35 \mathrm{eV}$. High spatial resolution MIR spectroscopy of eight local AGNs indicated that the [S IV] emission is unresolved at a scale of 100 pc (Hönig et al. 2008), while near-IR integral-field-unit data of Circinus showed that most of the [Si VI] and [Ca VIII] emission is unresolved at $4 \mathrm{pc}$ (Müller Sánchez et al. 2006). This velocity dispersion excess is seen even for the [O III] $5007 \AA$ line, which is resolved for all systems in our sample. Greene \& Ho (2005) also reported such an excess in a large flux-limited sample of AGNs observed with SDSS.

Another trend known from optical NLR studies is that the widths of the lines increase with their own luminosities (Phillips et al. 1983; Whittle 1985, 1992b). We reproduce this result for the MIR fine-structure lines that we examined in Figure 9. The velocity dispersion correlates roughly as $L^{0.15}$ based on, e.g., the $[\mathrm{Ne} \mathrm{V}]$ measurements. A higher dependence of $\sigma$ on $L$ is plausible when taking into account the upper limits of $\sigma$. An 


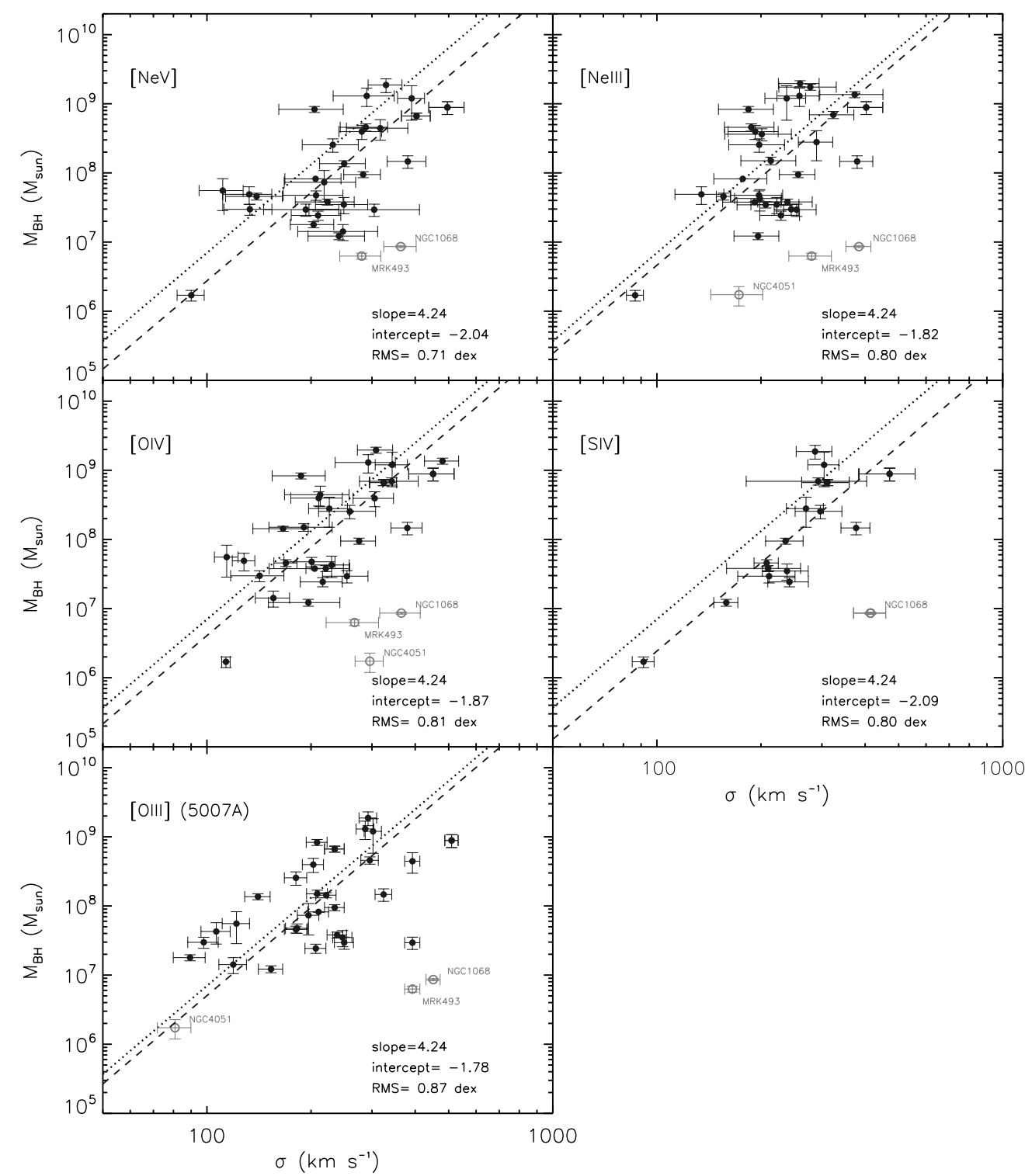

Figure 10. $M_{\mathrm{BH}}$ vs. NLR velocity dispersion. Open circles correspond to sources included in the rms computation, but excluded from the fit due to the lack of corresponding Spitzer data for the left-hand side of the relation. The best-fit solution to all other data points is presented with a dashed line. The dotted line corresponds to the Gültekin et al. (2009) relation, thought appropriate for the velocity dispersion of the stars in the bulge.

exact determination of this slope will require the use of a fluxlimited sample of AGNs in the local universe.

\subsection{Line Profiles: Shifts and Asymmetries}

Changes in the profile moments of different lines can also be seen when the emission originates from gas out of dynamical equilibrium. Several of the 81 sources in our sample show signatures of outflowing (or inflowing) gas motions, such as an offset from systemic velocity. This offset often depends on the ionization potential of each ionic species. With increasing ionization potential, the lines trace clouds that are nearer to the $\mathrm{BH}$, and therefore more susceptible to outflows. We identified six sources in which the offset between the peak of the lowest ionization line available and the highest ionization line available exceeds Nyquist sampling of the resolution element, $\gtrsim 250 \mathrm{~km} \mathrm{~s}^{-1}$. Namely, these are the type 1 AGNs $3 \mathrm{C} 273$ and IRAS13342+3932, and the type 2 AGNs IRAS05189-2524, IRAS15001+1433, IRAS23060+0505, and Mrk 609 (see also Spoon et al. 2009; Spoon \& Holt 2009). Line shifts below
Nyquist sampling of the instrumental resolution, yet above the wavelength calibration uncertainty $\left(50 \mathrm{~km} \mathrm{~s}^{-1}\right)$, could also be theoretically observed. They could be considered real if they systematically increased as a function of the ionization potential of the ionic species. Only one source ${ }^{9}$ satisfies this criterion, Mrk 1457. Its [Ne v] and [Ne II] lines are offset by $100 \mathrm{~km} \mathrm{~s}^{-1}$.

Asymmetric wings are found in $\sim 1 / 5$ th of the sources with resolved profiles. We consider such wings to be reliable only when they are detected in two or more lines. Similar [O III] $5007 \AA$ and [Ne III] or [Ne v] wings are observed, for example, in PG1351+640, PG1411+442, and PG1440+356. Likewise, an agreement of the [O III] and [O IV] $25.89 \mu \mathrm{m}$ line profiles is found for 3C273, IRAS13451+1232, IRAS15001+1433, Mrk 1014, NGC 4235, and NGC 7674.

\footnotetext{
9 Other sources, such as NGC 2623, NGC 7469, Arp148, and PG1211+143, could also have outflows of ionized gas, which need to be proven with spectroscopy of higher resolution than that of IRS. The blue wings of [ $\mathrm{Ne} \mathrm{V}]$ and [O IV] in NGC 6240 are also redshifted with respect to [Ne II] and [Ne III], but their overall line shifts are hard to determine as these lines are blended with $[\mathrm{Cl} \mathrm{II}]$ and [Fe II] (see also Armus et al. 2006).
} 


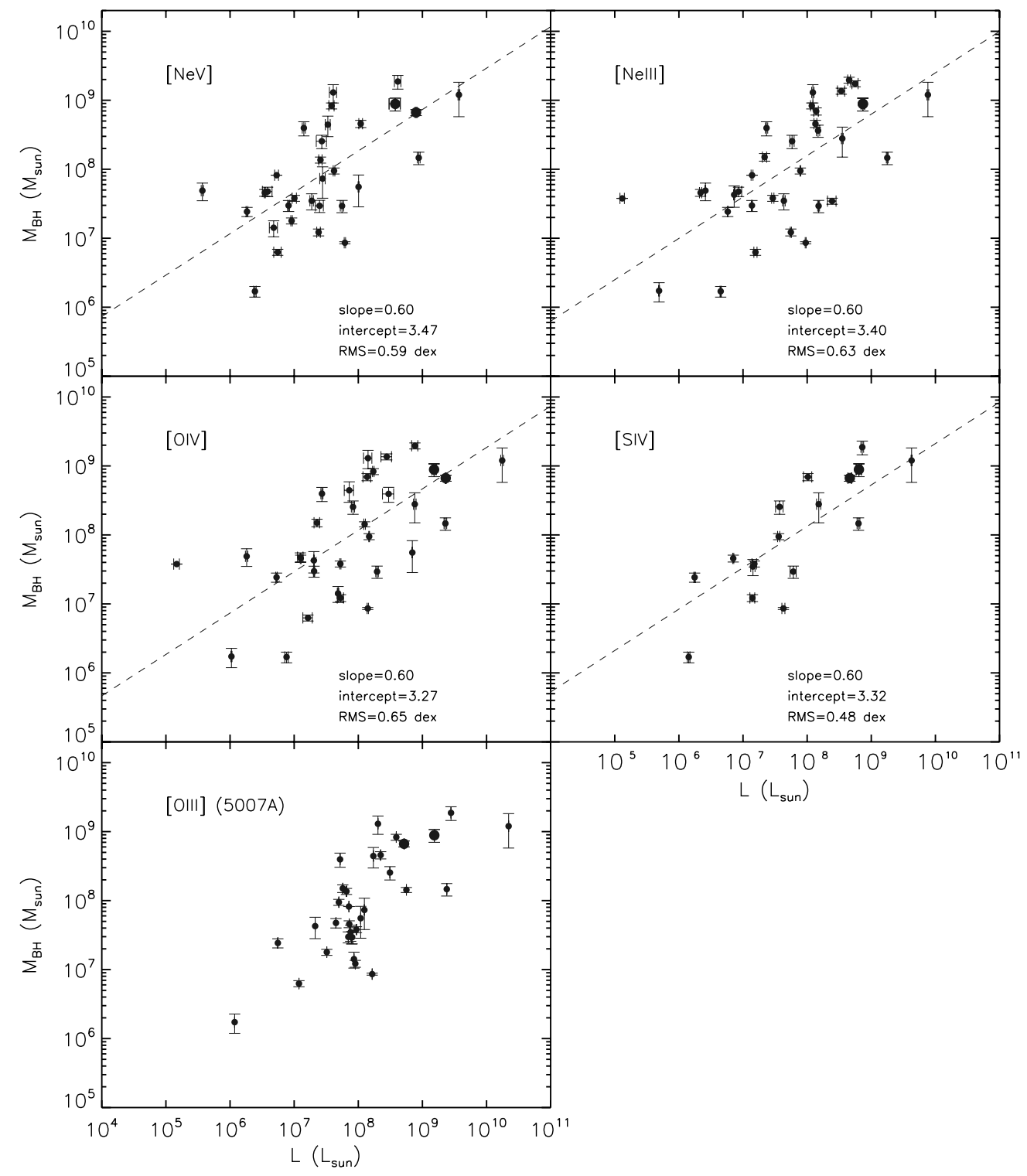

Figure 11. $M_{\mathrm{BH}}$ vs. narrow-line luminosity relation.

Specifically, IRAS13451+1232 is a merging system with two nuclei separated by $\sim 5 \mathrm{kpc}$ (e.g., Axon et al. 2000; Dasyra et al. 2006a). The velocity of the secondary [O IV] peak, at $\sim-1000 \mathrm{~km} \mathrm{~s}^{-1}$, is comparable to the velocities of the outflowing $\left[\mathrm{O}_{\mathrm{I}}\right]$ and $[\mathrm{O} \mathrm{III}]$ gas components that are seen in optical spectroscopy and that are associated with the nucleus responsible for the radio-jet emission (Holt et al. 2003).

\section{DISCUSSION}

\subsection{A Three-dimensional Plane Connecting $M_{B H}$ and the $L$ and $\sigma$ of the NLR?}

By performing a similar profile analysis of the $[\mathrm{Ne} \mathrm{V}]$ and [O IV] lines, restricted only to AGNs with direct $M_{\mathrm{BH}}$ measurements from reverberation experiments (Peterson et al. 2004), we previously demonstrated that the NLR velocity dispersion correlates with the mass of its central BH (Dasyra et al. 2008). We now further populate this relation using data from the full Spitzer archive, complementing them with ISO SWS data to cover the parameter space $\sigma \lesssim 200 \mathrm{~km} \mathrm{~s}^{-1}$. The $M_{\mathrm{BH}}$ values for this expanded sample include single-epoch optical spec- troscopic estimates for type 1 AGNs, as well as direct $M_{\mathrm{BH}}$ measurements for a few type 2 AGNs (see Section 2; Tables 3 and 4).

The fit to these data (Figure 10) was performed with the IDL routine MPFITFUN. Given the sparsity of data at the low $\sigma$ end, we opted for a linear fit of fixed slope in logarithmic space. The slope was set to 4.24 , which is identical to that of the stellar relation (Ferrarese \& Merritt 2000; Gebhardt et al. 2000) as recently revised by Gültekin et al. (2009). While the rms scatter was computed using all available data points, the bestfit solution was computed using only Spitzer observations of sources with $M_{\mathrm{BH}}>10^{7} M_{\odot}$ or $I S O$ observations, as in Dasyra et al. (2008). The reason why we excluded Spitzer data sets for $M_{\mathrm{BH}}<10^{7} M_{\odot}$ is that they would introduce a bias toward high intercept values: the resolution of IRS is insufficient to resolve lines on the left-hand side of the $M_{\mathrm{BH}}-\sigma$ relation below this threshold. This excludes the type 2 AGNs NGC 1068, and the narrow-line Seyfert 1 galaxies (NLS1s) Mrk 493 and NGC 4051 that have high $\sigma$ values for their $\mathrm{BH}$ masses. The existence of such outliers nonetheless suggests that $\sigma$ could fail as a proxy of $M_{\mathrm{BH}}$, as it is also known for the BLR gas (e.g., Vestergaard \& 

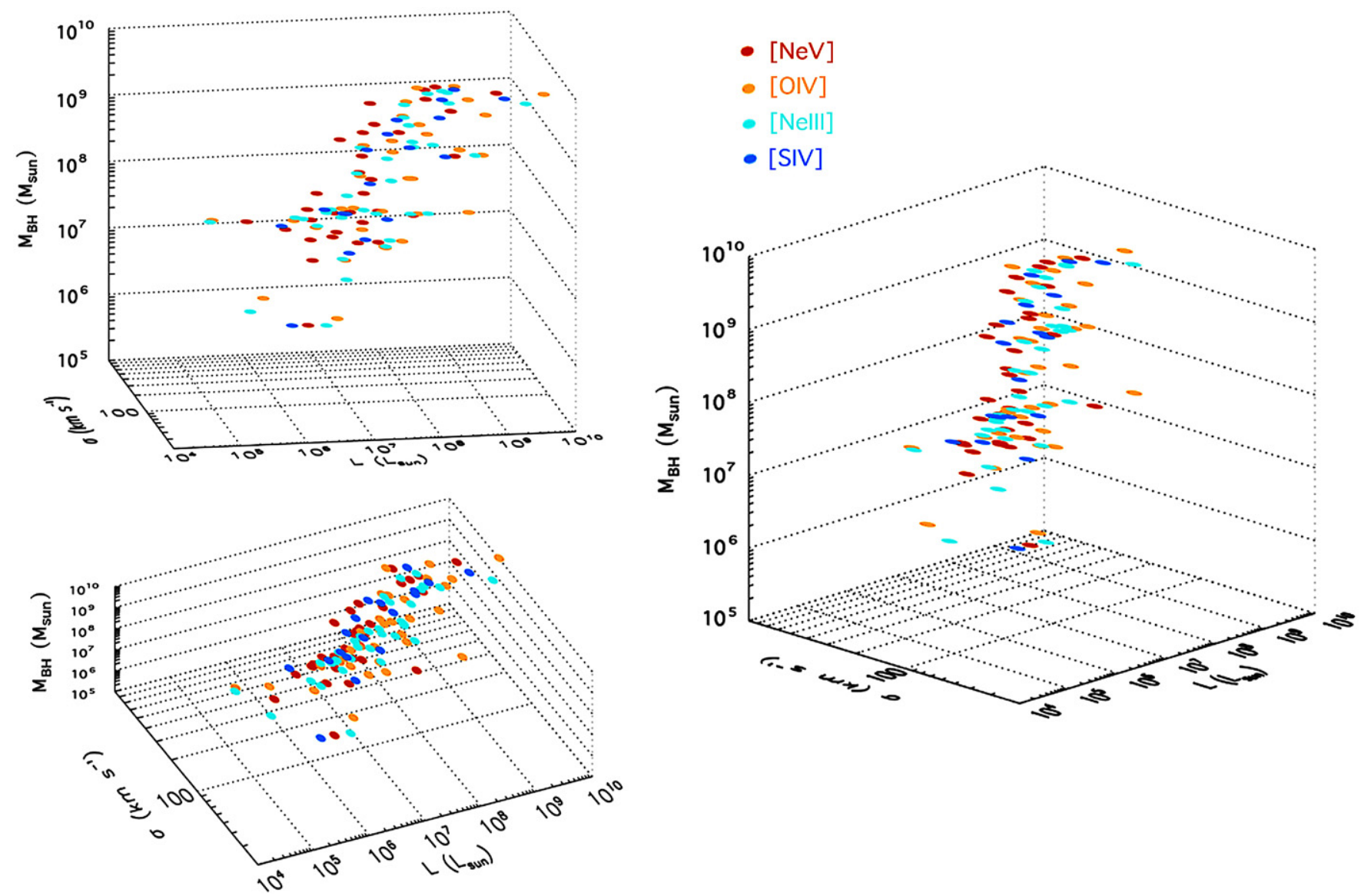

Figure 12. Different views of the plane suggested to connect $M_{\mathrm{BH}}$, and the velocity dispersion and luminosity of AGN narrow lines in the MIR.

Peterson 2006). On the other hand, such sources are not outliers in the relation connecting $M_{\mathrm{BH}}$ to the luminosity of the NLR lines (Figure 11).

The multiple relations between $M_{\mathrm{BH}}$ and $\sigma, \sigma$ and $L$, and $M_{\mathrm{BH}}$ and $L$ could be suggestive of a plane connecting these three parameters (Figure 12), which we fit using the equation

$$
\log \left(M_{\mathrm{BH}}\right)=\alpha \log (\sigma)+\beta \log (L)+\gamma,
$$

where $\alpha, \beta$, and $\gamma$ are constants. We find that the best-fit-solution coefficients correspond to $\alpha=0.9$ and $\beta=0.5$, when averaged over all MIR lines. Given the $L$ and $\sigma^{\sim 7}$ proportionality shown in Figure 9, this result roughly reproduces the $M_{\mathrm{BH}} \sim \sigma^{4}$ relation. In Figure 13, we present the plane equation for each line, when fixing the $\log (\sigma)$ and $\log (L)$ slopes to their average values for simplicity. ${ }^{10}$ We find that the use of a plane equation minimizes the scatter of the NLR-based $M_{\mathrm{BH}}$ estimates from their actual values. The improvement primarily originates from the correction of the $M_{\mathrm{BH}}-\sigma$ relation outliers at its low- $M_{\mathrm{BH}}$ and high- $\sigma$ end.

\subsection{The Origin of the Scaling Relations between the NLR Gas Properties and $M_{B H}$}

The physical interpretation of the suggested plane linking the NLR line luminosity, velocity dispersion, and the BH mass, varies depending upon the assumed kinematics and distribution

\footnotetext{
10 We have adapted this approach throughout all fitting procedures in this work (see also Figures 10 and 11) to facilitate comparisons of the rms scatter among the various relations, and to avoid biases related to the small galaxy sample that is used for their creation and that varies from line to line.
}

of the gas clouds. A plane equation can be meaningful for clouds on accelerated motions powered directly by the AGN via radiation pressure (Murayama \& Taniguchi 1998) acting mostly upon dust particles due to their high opacity (Dopita et al. 2002), magnetic fields related to jets (Whittle 1992c), and AGN-related winds. The winds can lead to either asymmetric outflows, identified in $7 \%$ of the systems in this sample, or to symmetric outflows that can be related to the AGN accretion disk (Crenshaw et al. 2003; Ho 2009). In this scenario, the measured gas velocity would be related to the fraction of the energy generated by the AGN that is deposited into the NLR gas. The luminosity would be a probe of the AGN accretion rate, since the luminosities of MIR NLR lines are known to be correlated with the optical, X-ray, and bolometric AGN luminosities (Schweitzer et al. 2006; Dasyra et al. 2008; Meléndez et al. 2008; Rigby et al. 2009; R. Mor et al. 2011, in preparation). A plane connecting $\sigma, L$, and $M_{\mathrm{BH}}$ would then suggest that the kinetic energy of the interstellar medium (ISM), as measured from the MIR lines, is directly related to the amount of material that is accreted onto the $\mathrm{BH}$ for a given $M_{\mathrm{BH}}$ value. An analogous idea was introduced by Merloni et al. (2003), who found a correlation between the mass of an accreting $\mathrm{BH}$ with its X-ray and radio luminosities.

Instead of responding to AGN feedback mechanisms, the gas clouds could be in virial motions that are dictated by the enclosed mass at the NLR radius $R$. For a geometric factor $f$ converting this total mass to the $\mathrm{BH}$ mass, the virial equation can be written as

$$
\log \left(M_{\mathrm{BH}}\right)=2 \log (\sigma)+\log (R)+\log (f)-\log (G),
$$




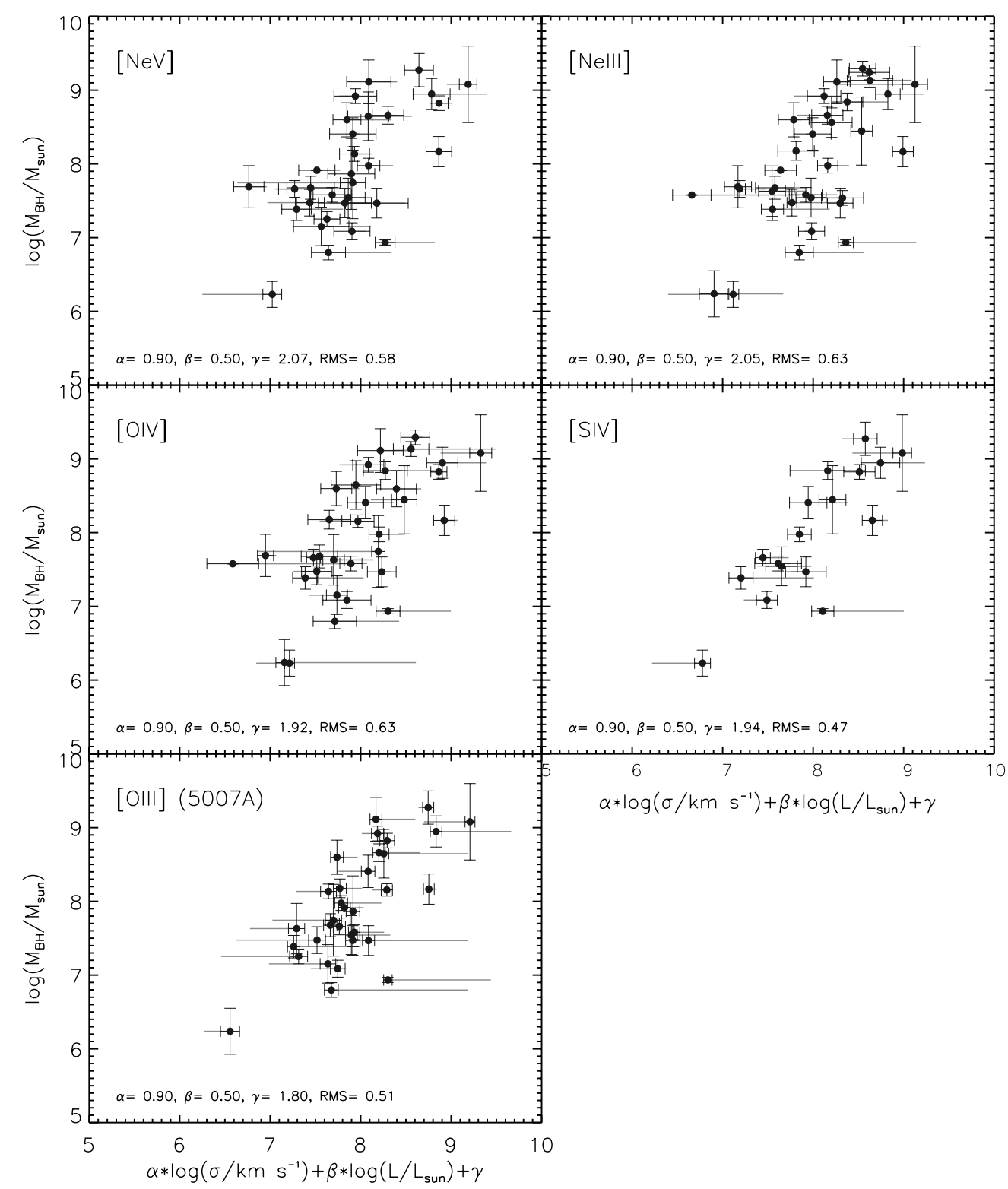

Figure 13. Linear combination, i.e., plane equation, of $\log (\sigma)$ and $\log (L)$ that describes $\log \left(M_{\mathrm{BH}}\right)$. The horizontal solid lines indicate where each source would have been located if its $M_{\mathrm{BH}}$ was estimated from the $M_{\mathrm{BH}}-\sigma$ relation (Figure 10) instead.

where $G$ is the gravitational constant. In this model, the NLR radius depends on the AGN luminosity alongside with the stellar mass and distribution in the AGN host galaxy. Equation (2) will take the form of Equation (1) if $R$ scales with the AGN luminosity as a power law of $L$, as found for the [O III] $5007 \AA$ line (Bennert et al. 2002; Schmitt et al. 2003). The hardness of the ionizing radiation together with the ionization fraction, the covering factor, and the density distribution of the clouds can also determine how far the AGN radiation reaches, illuminates, and photoionizes gas clouds. For example, the observed increase of $\sigma$ with $L$ can be driven either by a tendency for more massive BHs to reside in larger galaxies (Ho 2009) or by the ionization state of the NLR. Lines from ions of high ionization potential are thought to be tracing matter-bound clouds that are partially ionized (Murayama et al. 1998; Wilson et al. 1997). In the case of partially ionized clouds, an increase of the AGN luminosity can lead to an overall expansion of the NLR, while most of the ionization can still occur for clouds at small radii. The exact relation between $\sigma, L$, and $M_{\mathrm{BH}}$ will depend on the gas density distribution, which is encapsulated in the scaling factor $f$. The coefficients of the best-fit plane solution, $\alpha=0.9$ and $\beta=0.5$, suggest that $f$ is dropping with $\sigma$, corresponding to a lower scaling factor for denser gas within a fixed radius. If, however, the scaling factor $f$ was constant, $\alpha$ would be equal to 2 , and the best-fit plane solution would correspond to $\beta=0.4$. Such a solution could also be plausible. It would increase the rms scatter by a small amount, i.e., by only $0.01-0.02$ dex for the MIR lines and by 0.07 dex for [O III].

If the cloud kinematics cannot be approximated by either feedback-driven motions or by virial motions, Equation (1) might not provide a good means of estimating $M_{\mathrm{BH}}$. For cloud kinematics that are described by a combination of virial and accelerated motions, e.g., in a virialized NLR where radiation pressure is significant, $M_{\mathrm{BH}}$ is proportional to $\sigma^{2} R G^{-1}+a^{\prime} L$, where $a^{\prime}$ is a constant that depends on the column density and level of ionization of the clouds (Marconi et al. 2008; but see also Netzer \& Marziani 2010). Further investigation of the optimal description of $M_{\mathrm{BH}}$ from narrow-line properties will be tested 
Table 5

Estimates of $M_{\mathrm{BH}}$ in Type 2 AGNs

\begin{tabular}{|c|c|c|c|}
\hline Galaxy & $\begin{array}{c}M_{\mathrm{BH}}\left(\sigma_{*}^{4.24}\right) \\
\left(10^{7} M_{\odot}\right)\end{array}$ & $\begin{array}{c}M_{\mathrm{BH}}\left(\sigma_{\mathrm{NLR}}^{4.24}\right) \\
\left(10^{7} M_{\odot}\right)\end{array}$ & $\begin{array}{c}M_{\mathrm{BH}} \text { (Plane Equation) } \\
\left(10^{7} M_{\odot}\right)\end{array}$ \\
\hline 2MASXJ08035923+2345201 & 2.1 & 9.3 & 4.4 \\
\hline 2MASXJ08244333+2959238 & 0.9 & 17 & 7.5 \\
\hline 2MASXJ10181928+3722419 & 0.7 & 5.3 & 7.3 \\
\hline 2MASXJ12384342+0927362 & 23 & 19 & 16 \\
\hline 2MASXJ16164729+3716209 & 23 & 6.6 & 22 \\
\hline CGCG218-007 & 3.8 & 7.2 & 7.3 \\
\hline ESO103-G035 & $\ldots$ & 29 & 8.4 \\
\hline IRAS05189-2524 & 2.7 & 132 & 29 \\
\hline IRAS15001+1433 & 70 & 225 & 41 \\
\hline IRAS18325-5926 & $\ldots$ & 70 & 17 \\
\hline IRAS23060+0505 & $\ldots$ & 75 & 45 \\
\hline MCG-03-34-064 & $\ldots$ & 76 & 22 \\
\hline Mrk 1066 & $\ldots$ & 20 & 7.9 \\
\hline Mrk 1457 & 3.2 & 24 & 10 \\
\hline Mrk 273 & 31 & 82 & 28 \\
\hline Mrk 3 & $\ldots$ & 40 & 19 \\
\hline Mrk 463E & 5.5 & 26 & 36 \\
\hline Mrk 609 & 3.4 & 120 & 14 \\
\hline Mrk 622 & 2.5 & 76 & 9.0 \\
\hline NGC 1275 & 32 & 27 & 11 \\
\hline NGC 2622 & $\ldots$ & 18 & 8.2 \\
\hline NGC 2623 & 4.1 & 37 & 7.5 \\
\hline NGC 2639 & 10 & 80 & 4.7 \\
\hline NGC 3079 & 3.9 & 40 & 3.0 \\
\hline NGC 4507 & $\ldots$ & 41 & 8.4 \\
\hline NGC 5256 & 9.9 & 9.2 & 8.1 \\
\hline NGC 5506 & $\ldots$ & 8.8 & 6.0 \\
\hline NGC 5728 & $\ldots$ & 32 & 8.7 \\
\hline NGC 5929 & 1.7 & 11 & 3.0 \\
\hline NGC 6240 & 23 & 51 & 17 \\
\hline NGC 7172 & $\ldots$ & 9.5 & 4.0 \\
\hline NGC 7674 & $\ldots$ & 19 & 16 \\
\hline SBS1133+572 & 15 & 12 & 11 \\
\hline UGC 02608 & $\ldots$ & 6.0 & 11 \\
\hline UGC 5101 & 10 & 71 & 17 \\
\hline
\end{tabular}

Notes. The second column corresponds to the result of the stellar $M_{\mathrm{BH}}-\sigma$ relation. The third column presents the $M_{\mathrm{BH}}$ estimate using the $M_{\mathrm{BH}}-\sigma$ relation for the NLR, as found in Figure 10. The fourth column uses the plane equation presented in Figure 12. For the NLR gas-based estimates, we present the average value using all resolved lines. Formal, statistical $M_{\mathrm{BH}}$ uncertainties are of a factor of 0.5 dex. For individual sources the uncertainty can be higher, typically within an order of magnitude.

on large, flux-limited samples of AGNs with multiwavelength data sets. This will be the focus of a forthcoming paper using SDSS data.

\subsection{Black Hole Mass Estimates in Obscured AGNs}

Given that we see no significant difference in the NLR properties of type 1 and type 2 AGNs at a given $L$ or $\sigma_{*}$ (see also Pereira-Santaella et al. 2010), we applied the relations presented earlier in this work to type 2 AGNs for which such an analysis is presently possible from Spitzer data. Only four of these sources have other, direct $M_{\mathrm{BH}}$ measurements (Table 2). The comparison between the $M_{\mathrm{BH}}$ estimates from the stellar $M_{\mathrm{BH}}-\sigma_{*}$ relation, the $M_{\mathrm{BH}}-\sigma$ relation for the NLR gas, and the best-fit linear combination of $\log (\sigma)$ and $\log (L)$ are presented in Table 5. For either computation based on the NLR gas, the result is averaged over all available MIR lines. We find that the median ratio of $M_{\mathrm{BH}}$ as estimated from the plane equation over $M_{\mathrm{BH}}$ as estimated from the stellar velocity dispersion is 1.8 . On the other hand, the median ratio of $M_{\mathrm{BH}}$ as estimated from the NLR gas velocity dispersion over $M_{\mathrm{BH}}$ as estimated from the stellar velocity dispersion is much higher, 6.5. The difference seen when folding a luminosity dependence on the computation of $M_{\mathrm{BH}}$ is largest for the IR-bright galaxies IRAS05189-2524, IRAS15001+1433, and Mrk609, which have gas motions that are predominantly out of dynamical equilibrium.

\section{CONCLUSIONS}

We queried the full Spitzer archive for high-resolution IRS spectra of type 1 AGNs, with BLR-based $M_{\mathrm{BH}}$ estimates from optical spectroscopy, and type 2 AGNs. We analyzed the spectra of 298 objects, which we combined with ISO spectra of 6 more AGNs, aiming to study the fundamental properties of the NLR in the IR, and to calibrate its fine-structure-line widths and luminosities to the mass of the central black hole. We found the following.

1. All of the [Ne v], [O IV], [Ne III], and [S IV] line luminosities are well correlated with each other (with an rms scatter of $\sim 0.2$ dex). Given the high ionization potential of Ne IV to $\mathrm{Ne} \mathrm{v}$, their emission is attributed to (further) photoionization of ions by the AGN. Thus, all of these lines can be used to study the NLR kinematics.

2. The bulk of the silicates responsible for the $9.7 \mu \mathrm{m}$ absorption feature is likely to be located in a region smaller than the NLR. Otherwise, the luminosity of [S IV] at $10.51 \mu \mathrm{m}$ would not correlate equally well with that of all other IR narrow lines without an extinction correction in obscured AGNs.

3. Of the 304 sources in our sample, 81 had at least one [Ne v], [OIV], [NeIII], or [SIV] narrow line that was resolved. We find that, on average, the line widths increase with increasing ionization potential of the species that emit them in the range 22-97 eV. Different fine-structure lines probe different (locations within the) clouds, with highionization-potential ions being preferentially found nearer to the $\mathrm{BH}$.

4. No trend was observed between the line width and critical density for these transitions, indicating that the average density of the NLR gas is typically below $10^{4}$ hydrogen atoms per cubic centimeter.

5. The velocity dispersions of all MIR lines that we examined, as well as that of the [O III] $5007 \AA$ line, are systematically above the velocity dispersion of the stars in the AGN host galaxies. Moreover, the line widths increase with their own luminosities, as known from optical wavelengths.

6. The mass of the central $\mathrm{BH}$ correlates with the velocity dispersion and with the luminosity of the NLR lines. We speculate that these results could be suggestive of a three-dimensional plane, connecting $\log \left(M_{\mathrm{BH}}\right)$ to a linear combination of $\log (\sigma)$ and $\log (L)$. Such a plane equation could be meaningful for a virial distribution in which the NLR radius has a power-law dependence on the AGN luminosity, or for AGN-feedback driven motions.

7. Given that we find no significant differences in the NLR kinematic properties of type 1 and type 2 AGNs, we used the NLR gas properties as seen in the MIR to estimate the $\mathrm{BH}$ masses residing in 35 local obscured AGNs.

K.D. acknowledges support by the European Community through a Marie Curie Fellowship (PIEF-GA-2009-235038) 
awarded under the Seventh Framework Programme (FP7/20072013). The authors thank T. Boroson and B. Peterson for providing optical spectra to be compared with the MIR ones, and S. Collin for useful discussions. This work made use of the NASA/IPAC Extragalactic Database, and it was based on archival data obtained with the Spitzer Space Telescope, operated by the Jet Propulsion Laboratory, California Institute of Technology under a contract with NASA.

\section{REFERENCES}

Armus, L., Bernard-Salas, J., Spoon, H. W. W., et al. 2006, ApJ, 640, 204 Axon, D. J., Capetti, A., Fanti, R., et al. 2000, AJ, 120, 2284

Baldwin, J., Philips, M., \& Terlevich, R. 1981, PASP, 93, 5

Bassani, L., Dadina, M., Maiolino, R., et al. 1999, ApJS, 121, 473

Bennert, N., Barvainis, R., Henkel, C., \& Antonucci, R. 2009, ApJ, 695, 276

Bennert, N., Falcke, H., Schulz, H., Wilson, A. S., \& Wills, B. J. 2002, ApJ, 574, L105

Bentz, M. C., Denney, K. D., Cackett, E. M., et al. 2006, ApJ, 651, 775

Bentz, M. C., Walsh, J. L., Barth, A. J., et al. 2009, ApJ, 705, 199

Blandford, R. D., \& McKee, C. F. 1982, ApJ, 255, 419

Boroson, T. A., \& Green, R. F. 1992, ApJS, 80, 109

Cappellari, M., Neumayer, N., Reunanen, J., et al. 2009, MNRAS, 394, 660

Crenshaw, D. M., Kraemer, S. B., \& George, I. M. 2003, ARA\&A, 41, 117

Dasyra, K. M., Ho, L. C., Armus, L., et al. 2008, ApJ, 674, L9

Dasyra, K. M., Tacconi, L. J., Davies, R. I., et al. 2006a, ApJ, 638, 745

Dasyra, K. M., Tacconi, L. J., Davies, R. I., et al. 2006b, ApJ, 651, 835

Dasyra, K. M., Tacconi, L. J., Davies, R. I., et al. 2007, ApJ, 657, 102

Denney, K. D., Peterson, B. M., Pogge, R. W., et al. 2010, ApJ, 721, 715

Dopita, M. A., Groves, B. A., Sutherland, R. S., Binette, L., \& Cecil, G. 2002, ApJ, 572, 753

Falcon-Barroso, J., Bacon, R., Bureau, M., et al. 2006, MNRAS, 369, 529

Ferguson, J. W., Korista, K. T., Baldwin, J. A., \& Ferland, G. J. 1997, ApJ, 487, 122

Ferrarese, L., \& Merritt, D. 2000, ApJ, 539, L9

Filippenko, A. V., \& Halpern, J. P. 1984, ApJ, 285, 458

Gebhardt, K., Bender, R., Bower, G., et al. 2000, ApJ, 539, L13

Genzel, R., Eckart, A., Ott, T., \& Eisenhauer, F. 1997, MNRAS, 291, 219

Gilli, R., Comastri, A., \& Hasinger, G. 2007, A\&A, 463, 79

Greene, J. E., \& Ho, L. C. 2005, ApJ, 627, 721

Greenhill, L. J., Booth, R. S., Ellingsen, S. P., et al. 2003, ApJ, 590, 162

Groves, B. A., Dopita, M. A., \& Sutherland, R. S. 2004, ApJS, 153, 9

Gorjian, V., Cleary, K., Werner, M. W., \& Lawrence, C. R. 2007, ApJ, 655, L73

Gültekin, K., Richstone, D. O., Gebhardt, K., et al. 2009, ApJ, 698, 198

Hao, L., Strauss, M. A., Fan, X., et al. 2005, AJ, 129, 1795

Harms, R. J., Ford, H. C., Tsvetanov, Z. I., et al. 1994, ApJ, 435, L35

Heckman, T. M., van Breugel, W. J. M., Miley, G. K., \& Butcher, H. R. 1983, AJ, 88,1077

Herrnstein, J. R., Moran, J. M., Greenhill, L. J., \& Trotter, A. S. 2005, ApJ, 629, 719

Hinz, J. L., \& Rieke, G. H. 2006, ApJ, 646, 872

Ho, L. C. 2007, ApJ, 668, 94

Ho, L. C. 2009 , ApJ, 699, 638

Ho, L. C., Filippenko, A. V., \& Sargent, W. L. W. 1997a, ApJS, 112, 315

Ho, L. C., Filippenko, A. V., \& Sargent, W. L. W. 1997b, ApJ, 487, 568

Ho, L. C., \& Kim, M. 2009, ApJS, 184, 398

Holt, J., Tadhunter, C. N., \& Morganti, R. 2003, MNRAS, 342, 227

Hönig, S. F., Smette, A., Beckert, T., et al. 2008, A\&A, 485, L21

Houck, J., Roellig, T. L., van Cleve, J., et al. 2004, ApJS, 154, 18

Kauffmann, G., Heckman, T. M., Tremonti, C., et al. 2003, MNRAS, 346, 1055

Kaspi, S., Smith, P. S., Netzer, H., et al. 2000, ApJ, 533, 631

Kaspi, S., Brandt, W. N., Maoz, D., et al. 2007, ApJ, 659, 997

Kewley, L. J., Groves, B., Kauffmann, G., \& Heckman, T. 2006, MNRAS, 372, 961
Kim, M., Ho, L. C., Peng, C. Y., et al. 2008, ApJ, 687, 767

Lacy, M., Petric, A. O., Sajina, A., et al. 2007, AJ, 133, 186

Laor, A. 2007, in ASP Conf. Ser. 373, The Central Engine of Active Galactic

Nuclei, ed. L. C. Ho \& J.-M. Wang (San Francisco, CA: ASP), 384

Liu, X., Zakamska, N. L., Greene, J. E., et al. 2009, ApJ, 702, 1098

Lodato, G., \& Bertin, G. 2003, A\&A, 398, 517

Macchetto, F., Marconi, A., Axon, D. J., et al. 1997, ApJ, 489, 579

Marconi, A., Axon, D. J., Maiolino, R., et al. 2008, ApJ, 678, 693

Marconi, A., Risaliti, G., Gilli, R., et al. 2004, MNRAS, 351, 169

Marziani, P., Sulentic, J. W., Zamanov, R., et al. 2003, ApJS, 145, 199

Meléndez, M., Kraemer, S. B., Armentrout, B. K., et al. 2008, ApJ, 682, 94

Merloni, A., Heinz, S., \& di Matteo, T. 2003, MNRAS, 345, 1057

Merloni, A., Rudnick, G., \& Di Matteo, T. 2004, MNRAS, 354, L37

Miyoshi, M., Moran, J., Herrnstein, J., et al. 1995, Nature, 373, 127

Mulchaey, J. S., Koratkar, A., Ward, M. J., et al. 1994, ApJ, 436, 586

Müller Sánchez, F., Davies, R. I., Eisenhauer, F., et al. 2006, A\&A, 454, 481

Murayama, T., \& Taniguchi, Y. 1998, ApJ, 497, L9

Murayama, T., Taniguchi, Y., \& Iwasawa, K. 1998, AJ, 115, 460

Nelson, C. H. 2000, ApJ, 554, L91

Nelson, C. H., Green, R. F., Bower, G., Gebhardt, K., \& Weistrop, D. 2004, ApJ, 615,652

Nelson, C. H., \& Whittle, M. 1996, ApJ, 465, 96

Netzer, H. 2004, IAUS, 222, 389

Netzer, H., \& Marziani, P. 2010, ApJ, 724, 318

Netzer, H., \& Trakhtenbrot, B. 2007, ApJ, 654, 754

Neumayer, N., Cappellari, M., Reunanen, J., et al. 2007, ApJ, 671, 1329

Oliva, E., Salvati, M., Moorwood, A. F. M., \& Marconi, A. 1994, A\&A, 288, 457

Onken, C. A., Ferrarese, L., Merritt, D., et al. 2004, ApJ, 615, 645

Pahre, M. A. 1999, ApJS, 124, 127

Pereira-Santaella, M., Diamond-Stanic, A. M., Alonso-Herrero, A., \& Rieke, G. H. 2010, ApJ, 725, 2270

Peterson, B. M. 1993, PASP, 105, 247

Peterson, B. M., Ferrarese, L., Gilbert, K. M., et al. 2004, ApJ, 613, 682

Phillips, M. M., Charles, P. A., \& Baldwin, J. A. 1983, ApJ, 266, 485

Reyes, R., Zakamska, N. L., Strauss, M. A., et al. 2008, AJ, 136, 2373

Rigby, J. R., Diamond-Stanic, A. M., \& Aniano, G. 2009, ApJ, 700, 1878

Shields, G. A., Gebhardt, K., Salviander, S., et al. 2003, ApJ, 583, 124

Schmitt, H. R., Antonucci, R. R. J., Ulvestad, J. S., et al. 2001, ApJ, 555, 663

Schmitt, H. R., Donley, J. L., Antonucci, R. R. J., Hutchings, J. B., \& Kinney, A. L. 2003, ApJS, 148, 32

Schweitzer, M., Groves, B., Netzer, H., et al. 2008, ApJ, 679, 101

Schweitzer, M., Lutz, D., Sturm, E., et al. 2006, ApJ, 649, 79

Soifer, B. T., Neugebauer, G., Matthews, K., Egami, E., \& Weinberger, A. J. 2002, AJ, 124, 2980

Spinelli, P. F., Storchi-Bergmann, T., Brandt, C. H., \& Calzetti, D. 2006, ApJS, 166,498

Spoon, H. W. W., Armus, L., Marshall, J. A., et al. 2009, ApJ, 693, 1223

Spoon, H. W. W., \& Holt, J. 2009, ApJ, 702, L42

Sturm, E., Lutz, D., Verma, A., et al. 2002, A\&A, 393, 821

Tacconi, L. J., Genzel, R., Lutz, D., et al. 2002, ApJ, 580, 73

Tecza, M., Genzel, R., Tacconi, L. J., et al. 2000, ApJ, 537, 178

Tristram, K. R. W., Meisenheimer, K., Jaffe, W., et al. 2007, A\&A, 474, 837

Turner, T. J., George, I. M., Nandra, K., \& Mushotzky, R. F. 1997, ApJS, 113, 23

Veilleux, S., Kim, D.-C., \& Sanders, D. B. 1999, ApJ, 522, 113

Veilleux, S., Kim, D.-C., Sanders, D. B., Mazzarella, J. M., \& Soifer, B. T. 1995, ApJS, 98, 171

Vestergaard, M., \& Peterson, B. M. 2006, ApJ, 641, 689

Whittle, M. 1985, MNRAS, 213, 1

Whittle, M. 1992a, ApJ, 79, 49

Whittle, M. 1992b, ApJ, 387, 109

Whittle, M. 1992c, ApJ, 387, 121

Wilson, A. S., Binette, L., \& Storchi-Bergmann, T. 1997, ApJ, 482, L131

Winter, L. M., Lewis, K. T., Koss, M., et al. 2010, ApJ, 710, 503

Zakamska, N., Strauss, M. A., Krolik, J. H., et al. 2003, AJ, 126, 2125 